$\begin{array}{lr}\text { Dept. of Math./CMA } & \text { University of Oslo } \\ \text { Pure Mathematics } & \text { No } 24\end{array}$

December 2009

\title{
Stochastic Differential Games in Insider Markets via Malliavin Calculus
}

\author{
Olivier Menoukeu Pamen ${ }^{* \dagger}$, Frank Proske ${ }^{\ddagger}$, Hassilah Binti Salleh ${ }^{\ddagger \S}$
}

December 22, 2009

\begin{abstract}
In this paper we use techniques of Malliavin calculus and forward integration to present a general stochastic maximum principle for anticipating stochastic differential equations driven by a Lévy type of noise. We apply our result to study a general stochastic differential game problem of an insider.
\end{abstract}

MSC2010: 60G51, 60H40, 60H10, 60HXX, 93E20

Key words: Malliavin calculus, maximum principle, jumps diffusion, stochastic control, insider information, forward integral, stochastic differential game.

\section{Introduction}

In real world, market agents have access to different levels of information and it is important to understand what value particular pieces of information have. This paper is devoted to the study of a class of two-player stochastic differential game in which the players have different information on the payoff. The different agents invest different amounts of capital in order to optimize their utility. We derive necessary and sufficient conditions for the existence of Nashequilibria for this game and characterize these for various levels of information asymmetry. The framework is the one of stochastic differential game with anticipative strategy sets.

In the following, let $\left\{B_{s}\right\}_{0 \leq s \leq T}$ be a Brownian motion and $\widetilde{N}(d z, d s)=N(d z, d s)-d s \nu(d z)$ be a compensated Poisson random measure associated with a Lévy process with Lévy measure $\nu$ on the (complete) filtered probability space $\left(\Omega, \mathcal{F},\left\{\mathcal{F}_{t}\right\}_{0 \leq t \leq T}, P\right)$. In the sequel, we assume that the Lévy measure $\nu$ fulfills

$$
\int_{\mathbb{R}_{0}} z^{2} \nu(d z)<\infty
$$

\footnotetext{
${ }^{*}$ School of Mathematics, University of the Witwatersrand, Private Bag 3, Wits 2050, Johannesburg, South Africa.

${ }^{\dagger}$ Programme in Advanced Mathematics of Finance, School of Computational and Applied Mathematics, University of the Witwatersrand, Private Bag 3, Wits 2050, Johannesburg, South Africa.

${ }^{\ddagger}$ CMA, Department of Mathematics, University of Oslo, P.O. Box 1053 Blindern, N-316 Oslo, Norway.

${ }^{\S}$ Department of Mathematics, Universiti Malaysia Terengganu, 21030 Kuala Terengganu, Terengganu, Malaysia.

Email: Olivier.MenoukeuPamen@wits.ac.za, proske@math.uio.no, hassilas@math.uio.no
} 
where $\mathbb{R}_{0}:=\mathbb{R} \backslash\{0\}$.

Suppose that the state process $X(t)=X^{(u)}(t, \omega) ; t \geq 0, \omega \in \Omega$ is a controlled Itô-Lévy process in $\mathbb{R}$ of the form:

$$
\left\{\begin{aligned}
d^{-} X(t)= & b\left(t, X(t), u_{0}(t), \omega\right) d t+\sigma\left(t, X(t), u_{0}(t), \omega\right) d^{-} B(t) \\
& +\int_{\mathbb{R}_{0}} \gamma\left(t, X(t), u_{0}(t), u_{1}(t, z), z, \omega\right) \widetilde{N}\left(d z, d^{-} t\right) ; \\
X(0)= & x \in \mathbb{R}
\end{aligned}\right.
$$

Where the coefficients $b:[0, T] \times \mathbb{R} \times U \times \Omega \longrightarrow \mathbb{R}, \sigma:[0, T] \times \mathbb{R} \times U \times \Omega \longrightarrow \mathbb{R}$, and $\gamma:[0, T] \times \mathbb{R} \times U \times K \times \mathbb{R}_{0} \times \Omega \longrightarrow \mathbb{R}$ are measurable functions, where $U \subset \mathbb{R}^{2}, K \subset \mathbb{R} \times \mathbb{R}_{0}$ are given open convex sets. Here we consider filtrations $\left\{\mathcal{G}_{t}^{i}\right\}_{t \in[0, T]}, i=1,2$ such that

$$
\mathcal{F}_{t} \subset \mathcal{G}_{t}^{i} \subset \mathcal{F}_{T}, \quad t \in[0, T], \quad i=1,2,
$$

representing the information available to the controller at time $t$.

Since $B(t)$ and $\tilde{N}(d z, d t)$ need not to be a semimartingale with respect to $\left\{\mathcal{G}_{t}^{i}\right\}_{t \geq 0}, i=1,2$, the two last integrals in (1.1) are anticipating stochastic integrals that we interpret as forward integrals.

The control processes $u_{0}(t)$ and $u_{1}(t, z)$ with values in given open convex sets $U$ and $K$ respectively for a.a $t \in[0, T], z \in \mathbb{R}_{0}$ are called admissible controls if (1.1) has a unique (strong) solution $X=X^{\left(u_{0}, u_{1}\right)}$ such that the components of $u_{0}(\cdot)$ and $u_{1}(\cdot, \cdot)$ are adapted to the considered filtrations $\left\{\mathcal{G}_{t}^{1}\right\}_{t \in[0, T]}$ and $\left\{\mathcal{G}_{t}^{2}\right\}_{t \in[0, T]}$ respectively.

Let $f:[0, T] \times \mathbb{R} \times U \times K \times \Omega \longrightarrow \mathbb{R}$ and $g: \mathbb{R} \times \Omega \longrightarrow \mathbb{R}$ be given measurable functions and the given performance functionals for players are as follows:

$$
J_{i}\left(u_{0}, u_{1}\right):=E^{x}\left[\int_{0}^{T} f_{i}\left(t, X(t), u_{0}(t), u_{1}(t, z), \omega\right) \mu(d z) d t+g_{i}(X(T), \omega)\right], i=1,2,
$$

where $\mu$ is a measure on the given measurable space $\left(\Omega, \mathcal{F}_{T}\right)$ and $E^{x}=E_{P}^{x}=E$ denotes the expectation with respect to $P$ given that $X(0)=x$. Suppose that the controls $u_{0}(t)$ and $u_{1}(t, z)$ have the form

$$
\begin{aligned}
u_{0}(t) & =\left(\pi_{0}(t), \theta_{0}(t)\right) ; t \in[0, T], \\
u_{1}(t, z) & =\left(\pi_{1}(t, z), \theta_{1}(t)\right) ; t \in[0, T] \times \mathbb{R}_{0} .
\end{aligned}
$$

Let $\mathcal{A}_{\Pi}$ (respectively $\mathcal{A}_{\Theta}$ ) denote the given family of controls $\pi=\left(\pi_{0}, \pi_{1}\right)$ (respectively $\left.\theta=\left(\theta_{0}, \theta_{1}\right)\right)$ such that they are contained in the set of $\mathcal{G}_{t}^{1}$-adapted controls (respectively $\mathcal{G}_{t}^{2}$-adapted controls), 1.1 has a unique strong solution up to time $T$ and

$$
E^{x}\left[\int_{0}^{T}\left|f_{i}\left(t, X(t), u_{0}(t), u_{1}(t, z), \omega\right)\right| \mu(d z) d t+\left|g_{i}(X(T), \omega)\right|\right]<\infty, i=1,2 .
$$

The insider information non-zero-sum stochastic differential game problem we analyze is the following: 
Problem 1.1 Find $\left(\pi^{*}, \theta^{*}\right) \in \mathcal{A}_{\Pi} \times \mathcal{A}_{\Theta}$ (if it exists) such that

1. $J_{1}\left(\pi, \theta^{*}\right) \leq J_{1}\left(\pi^{*}, \theta^{*}\right)$ for all $\pi \in \mathcal{A}_{\Pi}$

2. $J_{2}\left(\pi^{*}, \theta\right) \leq J_{2}\left(\pi^{*}, \theta^{*}\right)$ for all $\theta \in \mathcal{A}_{\Theta}$

The pair $\left(\pi^{*}, \theta^{*}\right)$ is called a Nash Equilibrium (if it exists). The intuitive idea is that there are two players, Player I and Player II. While Player I controls $\pi$, Player II controls $\theta$. Each player is assumed to know the equilibrium strategies of the other players, and no player has anything to gain by changing only his or her own strategy (i.e., by changing unilaterally). Player I and Player II are in Nash Equilibrium if each player is making the best decision she can, taking into account the other player's decision. Note that since we allow $b, \sigma, \gamma$, $f$ and $g$ to be stochastic processes and since our controls are also $\mathcal{G}_{t}^{1}$-adapted (respectively $\mathcal{G}_{t}^{2}$-adapted), this problem is not of Markovian type and hence cannot be embedded into the framework of dynamic programming.

Our paper is inspired by ideas developed by Di Nunno et al in [10] and, An et al in [2], where the authors use Malliavin calculus to derive a general maximum principle for anticipative stochastic control and a general maximum principle for stochastic differential games with partial information, respectively. The paper focus on the conditions on the enlarged filtration to obtain the non-existence of an optimal insider game. Our paper covers the insider case in [11, since we include jumps in the risky asset model and we deal with controls being adapted to general supfiltrations of the underlying reference filtration. Moreover, our Malliavin calculus approach to stochastic differential games with insider information for Itô-Lévy processes allows for optimization of very general performance functionals. We apply our results to a worst case scenario portfolio problem in finance under additional information. We show that there does not exist a Nash-equilibrium for the insider. We prove that there exists a Nash-equilibrium insider consumption, and in some special cases the optimal solution can be expressed explicitly.

The paper is organized as follows: In Section 2, we recall some basic concepts of forward integration. In Section 3, we derive a general maximum principle for insider stochastic differential game control problem using Malliavin calculus. In Section 4, the Itô-Lévy processes are considered. Finally in Section 5 and 6, we apply our results to study optimal and competing-insider control problem and optimal and competing-insider consumption problem. The Appendix is devoted to the proof of our main Theorem (Theorem 3.2).

\section{Forward integrals}

In this Section we briefly review some basic concepts of forward integration theory and its relation to Malliavin calculus, which we will use in the forthcoming sections. We refer to [13, 18, 21, 22] and [6] for more information about these forward integrations. As for Malliavin calculus the reader may consult [8] or [17]. 


\subsection{Forward integral for $B(\cdot)$}

We recall the forward integral with respect to the Brownian motion. Let $B(t)$ be a Brownian motion on a filtered probability space $\left(\Omega, \mathcal{F}, \mathcal{F}_{t \geq 0}, P\right)$, and $T>0$ a fixed horizon.

Definition 2.1 Let $\phi:[0, T] \times \Omega \rightarrow \mathbb{R}$ be a measurable process. The forward integral of $\phi$ with respect to $B(\cdot)$ is defined by

$$
\int_{0}^{T} \phi(t, \omega) d^{-} B(t)=\lim _{\epsilon \rightarrow 0} \int_{0}^{T} \phi(t, \omega) \frac{B(t+\epsilon)-B(t)}{\epsilon} d t
$$

if the limit exist in probability, in which case $\phi$ is called forward integrable.

Note that if $\phi$ is càdlàg and forward integrable, then

$$
\int_{0}^{T} \phi(t, \omega) d^{-} B(t)=\lim _{\Delta t \rightarrow 0} \sum_{j} \phi\left(t_{j}\right) \Delta B\left(t_{j}\right) .
$$

where the sum is taken over the points of a finite partition of $[0, T]$.

Denote by $D_{t}$ the Malliavin derivative in the direction of $B(t)$ and by $\mathbb{D}_{1,2}^{B}$ the stochastic Sobolev space with the norm $\|\cdot\|_{1,2}$ given by

$$
\|F\|_{1,2}=\left(\|F\|_{L^{2}(\mu)}+E\left[\int_{0}^{T}\left|D_{t} F\right|^{2}\right]\right)^{\frac{1}{2}} .
$$

See [8] or [17] for definitions and further results.

Definition 2.2 Let $\mathcal{M}^{B}$ denote the set of stochastic functions $\phi:[0, T] \times \Omega \rightarrow \mathbb{R}$ such that:

1. $\phi \in L^{2}([0, T] \times \Omega), u(t) \in \mathbb{D}_{1,2}^{B}$ for almost all $t$ and satisfies

$$
\mathbb{E}\left(\int_{0}^{T}|\phi(t)|^{2} d t+\int_{0}^{T} \int_{0}^{T}\left|D_{u} \phi(t)\right|^{2} d u d t\right)<\infty .
$$

We will denoted by $\mathbb{L}^{1,2}[0, T]$ the class of such processes.

2. $\lim _{\epsilon \rightarrow 0} \frac{1}{\epsilon} \int_{u-\epsilon}^{u} \phi(t) d t=\phi(u)$ for a. $a u \in[0, T]$ in $\mathbb{L}^{1,2}[0, T]$,

3. $D_{t+} \phi(t):=\lim _{s \rightarrow t+} D_{s} \phi(t)$ exists in $L^{1}((0, T) \otimes \Omega)$ uniformly in $t \in[0, T]$.

We let $\mathbb{M}_{1,2}^{B}$ be the closure of the linear span of $\mathcal{M}^{B}$ with respect to the norm given by

$$
\|\phi\|_{\mathbb{M}_{1,2}^{B}}:=\|\phi\|_{\mathbb{L}^{1,2}[0, T]}+\left\|D_{t+} \phi(t)\right\|_{L^{1}((0, T) \otimes \Omega)}
$$

Then we have the relation between the forward integral and the Skorohod integral (see [15, 8] ): 
Lemma 2.3 If $\phi \in \mathbb{M}_{1,2}^{B}$ then it is forward integrable and

$$
\int_{0}^{T} \phi(t) d^{-} B(t)=\int_{0}^{T} \phi(t) \delta B(t)+\int_{0}^{T} D_{t+} \phi(t) d t .
$$

Moreover

$$
\mathbb{E}\left[\int_{0}^{T} \phi(t) d^{-} B(t)\right]=\mathbb{E}\left[\int_{0}^{T} D_{t+} \phi(t) d t\right] .
$$

Using (2.3) and the duality formula for the Malliavin derivative $D_{t}$ see e.g. [8], one deduces the following result.

Corollary 2.4 Suppose $\phi \in \mathbb{M}_{1,2}^{B}$ and $F \in \mathbb{D}_{1,2}^{B}$ then

$$
\begin{aligned}
\mathbb{E}\left[F \int_{0}^{T} \phi(t) d^{-} B(t)\right] & =\mathbb{E}\left[F \int_{0}^{T} \phi(t) \delta B(t)+F \int_{0}^{T} D_{t+} \phi(t) d t\right] \\
& =\mathbb{E}\left[\int_{0}^{T} \phi(t) D_{t} F d t+\int_{0}^{T} F D_{t+} \phi(t) d t\right]
\end{aligned}
$$

where $\int_{0}^{T} \phi(t) \delta B(t)$ denotes the Skorohod integral w.r.t $B(t)$.

\subsection{Forward integral for $\widetilde{N}(\cdot, \cdot)$}

We give the forward integral with respect to the compensated Poisson random measure $\widetilde{N}$.

Definition 2.5 The forward integral

$$
J(\phi):=\int_{0}^{T} \int_{\mathbb{R}_{0}} \phi(t, z) \tilde{N}\left(d z, d^{-} t\right),
$$

with respect to the Poisson random measure $\tilde{N}$, of a càdlàg stochastic function $\phi(t, z), t \in$ $[0, T], z \in \mathbb{R}$, with $\phi(t, z)=\phi(\omega, t, z), \omega \in \Omega$,is defined as

$$
J(\phi)=\lim _{m \rightarrow \infty} \int_{0}^{T} \int_{\mathbb{R}} \phi(t, z) 1_{U_{m}} \tilde{N}(d z, d t),
$$

if the limit exists in $L^{2}(\mathbb{P})$. Here $U_{m}, m=1,2, \cdots$, is an increasing sequence of compact sets $U_{m} \subseteq \mathbb{R} \backslash\{0\}$ with $\nu\left(U_{m}\right)<\infty$ such that $\lim _{m \rightarrow \infty} U_{m}=\mathbb{R} \backslash\{0\}$.

As in the Gaussian case we shall indicate by $D_{t, z}^{\widetilde{N}}=D_{t, z}$ the Malliavin derivative in the direction of $\widetilde{N}$ and by $\mathbb{D}_{1,2}^{\widetilde{N}}$ the corresponding Sobolev stochastic space, see [8].

Definition 2.6 Let $\mathcal{M}^{\widetilde{N}}$ denote the set of stochastic functions $\phi:[0, T] \times \mathbb{R} \times \Omega \rightarrow \mathbb{R}$ such that:

1. $\phi(t, z, \omega)=\phi_{1}(t, \omega) \phi_{2}(t, z, \omega)$ where $\phi_{1}(\omega, t) \in \mathbb{D}_{1,2}^{\widetilde{N}_{2}}$ is càdlàg and $\phi_{2}(\omega, t, z)$ is adapted such that

$$
\mathbb{E}\left[\int_{0}^{T} \int_{\mathbb{R}} \phi_{2}(t, z) \nu(d z) d t\right]<\infty,
$$


2. $D_{t+, z} \phi:=\lim _{s \rightarrow t+} D_{s, z} \phi$ exists in $L^{2}(\mathbb{P} \times \lambda \times \nu)$,

3. $\phi(t, z)+D_{t+, z} \phi(t, z)$ is Skorohod integrable.

We let $\mathbb{M}_{1,2}^{\widetilde{N}}$ be the closure of the linear span of $\mathcal{M}^{B}$ with respect to the norm given by

$$
\|\phi\|_{\mathbb{M}_{1,2}^{\tilde{N}}}:=\|\phi\|_{L^{2}(\mathbb{P} \times \lambda \times \nu)}+\left\|D_{t+, z} \phi(t, z)\right\|_{L^{2}(\mathbb{P} \times \lambda \times \nu)}
$$

Then we have the following relation between the forward and the Skorohod integrals (see [6, 8] ):

Lemma 2.7 If $\phi \in \mathbb{M}_{1,2}^{\widetilde{N}}$ then it is forward integrable and

$$
\int_{0}^{T} \int_{\mathbb{R}} \phi(t, z) \tilde{N}\left(d z, d^{-} t\right)=\int_{0}^{T} \int_{\mathbb{R}} D_{t+, z} \phi(t, z) \nu(d z) d t+\int_{0}^{T} \int_{\mathbb{R}}\left(\phi(t, z)+D_{t+, z} \phi(t, z)\right) \tilde{N}(d z, \delta t) .
$$

Moreover

$$
\mathbb{E}\left[\int_{0}^{T} \int_{\mathbb{R}} \phi(t, z) \widetilde{N}\left(d z, d^{-} t\right)\right]=\mathbb{E}\left[\int_{0}^{T} \int_{\mathbb{R}} D_{t+, z} \phi(t, z) \nu(d z) d t\right] .
$$

Then by (2.6) and duality formula for Skorohod integral for Poisson process see [8], we have Corollary 2.8 Suppose $\phi \in \mathbb{M}_{1,2}^{\widetilde{N}_{1}}$ and $F \in \mathbb{D}_{1,2}^{\widetilde{N}_{1}}$, then

$$
\begin{aligned}
\mathbb{E}\left[F \int_{0}^{T} \int_{\mathbb{R}} \phi(t, z) \tilde{N}\left(d z, d^{-} t\right)\right]= & \mathbb{E}\left[F \int_{0}^{T} \int_{\mathbb{R}} D_{t+, z} \phi(t, z) \nu(d z) d t\right] \\
& +\mathbb{E}\left[F \int_{0}^{T} \int_{\mathbb{R}}\left(\phi(t, z)+D_{t+, z} \phi(t, z)\right) \tilde{N}(d z, \delta t)\right] \\
= & \mathbb{E}\left[\int_{0}^{T} \int_{\mathbb{R}} \phi(t, z) D_{t, z} F \nu(d z) d t\right] \\
& +\mathbb{E}\left[\int_{0}^{T} \int_{\mathbb{R}}\left(F+D_{t, z} F\right) D_{t+, z} \phi(t, z) \nu(d z) d t\right] .
\end{aligned}
$$

\section{A stochastic maximum principle for insider stochastic dif- ferential games}

We now return to Problem 1.1 given in the introduction. We make the following assumptions:

1. The functions $b:[0, T] \times \mathbb{R} \times U \times \Omega \rightarrow \mathbb{R}, \sigma:[0, T] \times \mathbb{R} \times U \times \Omega \rightarrow \mathbb{R}, \gamma:[0, T] \times \mathbb{R} \times$ $U \times K \times \mathbb{R}_{0} \times \Omega \rightarrow \mathbb{R}, f:[0, T] \times \mathbb{R} \times U \times \Omega \rightarrow \mathbb{R}$ and $g: \mathbb{R} \times \Omega \rightarrow \mathbb{R}$ are contained in $C^{1}$ with respect to the arguments $x \in \mathbb{R}, u_{0} \in U$ and $u_{1} \in K$ for each $t \in[0, T]$ and a.a. $\omega \in \Omega$. 
2. For all $s, r, t \in(0, T), t \leq r$ and all bounded $\mathcal{G}_{t}^{2}$-measurable (respectively $\mathcal{G}_{t}^{1}$-measurable) random variables $\alpha=\alpha(\omega)$ (respectively $\xi=\xi(\omega)), \omega \in \Omega$, the controls $\beta_{\alpha}(s):=$ $\left(0, \beta_{\alpha}^{i}(s)\right)$ and $\eta_{\xi}(s):=\left(0, \eta_{\xi}^{i}(s)\right)$ for $i=1,2$ with

$$
\beta_{\alpha}^{i}(s):=\alpha^{i}(\omega) \chi_{[t, r]}(s), \quad 0 \leq s \leq T,
$$

respectively

$$
\eta_{\xi}^{i}(s):=\xi^{i}(\omega) \chi_{[t, r]}(s), \quad 0 \leq s \leq T
$$

belong to $\mathcal{A}_{\Pi}$ (respectively $\mathcal{A}_{\Theta}$ ). Also, we will denote the transposes of the vectors $\beta$ and $\eta$ by $\beta^{*}, \eta^{*}$ respectively.

3. For all $\pi, \beta \in \mathcal{A}_{\Pi}$ with $\beta$ bounded, there exists a $\delta_{1}>0$ such that

$$
\pi+y \beta \in \mathcal{A}_{\Pi}, \text { for all } y \in\left(-\delta_{1}, \delta_{1}\right)
$$

and such that the family

$$
\begin{aligned}
& \left\{\frac{\partial}{\partial x} f_{1}\left(t, X^{(\pi+y \beta, \theta)}(t), \pi+y \beta, \theta, z\right) \frac{d}{d y} X^{(\pi+y \beta, \theta)}(t)\right. \\
& \left.+\nabla_{\pi} f_{1}\left(t, X^{(\pi+y \beta, \theta)}(t), \pi+y \beta, \theta, z\right) \beta^{*}(t)\right\}_{y \in\left(-\delta_{1}, \delta_{1}\right)}
\end{aligned}
$$

is $\lambda \times \nu \times \mathbb{P}$-uniformly integrable and

$$
\left\{g^{\prime}\left(X^{(\pi+y \beta, \theta)}(T)\right) \frac{d}{d y} X^{(\pi+y \beta, \theta)}(T)\right\}_{y \in\left(-\delta_{1}, \delta_{1}\right)}
$$

is $\mathbb{P}$-uniformly integrable. Similarly, for all $\theta, \eta \in \mathcal{A}_{\Theta}$ with $\eta$ bounded, there exists a $\delta_{2}>0$ such that

$$
\theta+v \eta \in \mathcal{A}_{\Theta}, \text { for all } v \in\left(-\delta_{2}, \delta_{2}\right)
$$

and such that the family

$$
\begin{aligned}
& \left\{\frac{\partial}{\partial x} f_{2}\left(t, X^{(\pi, \theta+v \eta)}(t), \pi, \theta+v \eta, z\right) \frac{d}{d y} X^{(\pi, \theta+v \eta)}(t)\right. \\
& \left.+\nabla_{\theta} f_{2}\left(t, X^{(\pi, \theta+v \eta)}(t), \pi, \theta+v \eta, z\right) \eta^{*}(t)\right\}_{v \in\left(-\delta_{2}, \delta_{2}\right)}
\end{aligned}
$$

is $\lambda \times \nu \times \mathbb{P}$-uniformly integrable and

$$
\left\{g^{\prime}\left(X^{(\pi, \theta+v \eta)}(T)\right) \frac{d}{d y} X^{(\pi, \theta+v \eta)}(T)\right\}_{v \in\left(-\delta_{2}, \delta_{2}\right)}
$$

is $\mathbb{P}$-uniformly integrable.

4. For all $\pi, \beta \in \mathcal{A}_{\Pi}$ and $\theta, \eta \in \mathcal{A}_{\Theta}$ with $\beta, \eta$ bounded the processes

$$
Y(t)=Y_{\beta}(t)=\left.\frac{d}{d y} X^{(\pi+y \beta, \theta)}(t)\right|_{y=0}, \quad V(t)=V_{\eta}(t)=\left.\frac{d}{d v} X^{(\pi, \theta+v \eta)}(t)\right|_{v=0}
$$


exist and follow the SDE, respectively:

$$
\begin{aligned}
d Y_{\beta}^{\pi}(t)= & Y_{\beta}\left(t^{-}\right)\left[\frac{\partial}{\partial x} b\left(t, X(t), \pi_{0}(t), \theta_{0}(t)\right) d t+\frac{\partial}{\partial x} \sigma\left(t, X(t), \pi_{0}(t), \theta_{0}(t)\right) d^{-} B(t)\right. \\
& \left.+\int_{\mathbb{R}_{0}} \frac{\partial}{\partial x} \gamma\left(t, X\left(t^{-}\right), \pi_{0}(t), \pi_{1}\left(t^{-}, z\right), \theta_{0}\left(t^{-}\right), \theta_{1}\left(t^{-}, z\right), z\right) \tilde{N}\left(d z, d^{-} t\right)\right] \\
& +\beta^{*}(t)\left[\nabla_{\pi} b\left(t, X(t), \pi_{0}(t), \theta_{0}(t)\right) d t+\nabla_{\pi} \sigma\left(t, X(t), \pi_{0}(t), \theta_{0}(t)\right) d^{-} B(t)\right. \\
& \left.+\int_{\mathbb{R}_{0}} \nabla_{\pi} \gamma\left(t, X\left(t^{-}\right), \pi_{0}(t), \pi_{1}\left(t^{-}, z\right), \theta_{0}\left(t^{-}\right), \theta_{1}\left(t^{-}, z\right), z\right) \tilde{N}\left(d z, d^{-} t\right)\right] \\
Y(0)= & 0
\end{aligned}
$$

and

$$
\begin{aligned}
d V_{\eta}^{\theta}(t)= & V_{\eta}\left(t^{-}\right)\left[\frac{\partial}{\partial x} b\left(t, X(t), \pi_{0}(t), \theta_{0}(t)\right) d t+\frac{\partial}{\partial x} \sigma\left(t, X(t), \pi_{0}(t), \theta_{0}(t)\right) d^{-} B(t)\right. \\
& \left.+\int_{\mathbb{R}_{0}} \frac{\partial}{\partial x} \gamma\left(t, X\left(t^{-}\right), \pi_{0}(t), \pi_{1}\left(t^{-}, z\right), \theta_{0}\left(t^{-}\right), \theta_{1}\left(t^{-}, z\right), z\right) \tilde{N}\left(d z, d^{-} t\right)\right] \\
& +\eta^{*}(t)\left[\nabla_{\theta} b\left(t, X(t), \pi_{0}(t), \theta_{0}(t)\right) d t+\nabla_{\theta} \sigma\left(t, X(t), \pi_{0}(t), \theta_{0}(t)\right) d^{-} B(t)\right. \\
& \left.+\int_{\mathbb{R}_{0}} \nabla_{\theta} \gamma\left(t, X\left(t^{-}\right), \pi_{0}(t), \pi_{1}\left(t^{-}, z\right), \theta_{0}\left(t^{-}\right), \theta_{1}\left(t^{-}, z\right), z\right) \tilde{N}\left(d z, d^{-} t\right)\right] \\
V(0)= & 0
\end{aligned}
$$

5. Suppose that for all $\pi \in \mathcal{A}_{\Pi}$ and $\theta \in \mathcal{A}_{\Theta}$ the following processes

$$
\begin{aligned}
& K_{i}(t):= g_{i}^{\prime}(X(T))+\int_{t}^{T} \int_{\mathbb{R}_{0}} \frac{\partial}{\partial x} f_{i}\left(s, X(s), \pi, \theta, z_{1}\right) \mu\left(d z_{1}\right) d s \\
& D_{t} K_{i}(t):=D_{t} g_{i}^{\prime}(X(T))+\int_{t}^{T} D_{t} \frac{\partial}{\partial x} f_{i}\left(s, X(s), \pi, \theta, z_{1}\right) \mu\left(d z_{1}\right) d s \\
& D_{t, z} K_{i}(t):=D_{t, z} g_{i}^{\prime}(X(T))+\int_{t}^{T} \int_{\mathbb{R}_{0}} D_{t, z} \frac{\partial}{\partial x} f_{i}\left(s, X(s), \pi, \theta, z_{1}\right) \mu\left(d z_{1}\right) d s \\
& H_{i}^{0}(s, x, \pi, \theta):= K_{i}(s)\left(b\left(s, x, \pi_{0}, \theta_{0}\right)+D_{s+} \sigma\left(s, x, \pi_{0}, \theta_{0}\right)\right. \\
&\left.+\int_{\mathbb{R}_{0}} D_{s+, z} \gamma(s, x, \pi, \theta, z) \nu(d z)\right)+D_{s} K(s) \sigma\left(s, x, \pi_{0}, \theta_{0}\right) \\
&+\int_{\mathbb{R}_{0}} D_{s, z} K(s)\left\{\gamma(s, x, \pi, \theta, z)+D_{s+, z} \gamma(s, x, \pi, \theta, z)\right\} \nu(d z)
\end{aligned}
$$




$$
\begin{aligned}
G(t, s):= & \exp \left[\int_{t}^{s}\left\{\frac{\partial b}{\partial x}\left(r, X(r), \pi_{0}(r), \theta_{0}(r)\right)-\frac{1}{2}\left(\frac{\partial \sigma}{\partial x}\right)^{2}\left(r, X(r), \pi_{0}(r), \theta_{0}(r)\right)\right\} d r\right. \\
& +\int_{t}^{s} \frac{\partial \sigma}{\partial x}\left(r, X(r), \pi_{0}(r), \theta_{0}(r)\right) d^{-} B(r) \\
& +\int_{t}^{s} \int_{\mathbb{R}_{0}}\left\{\ln \left(1+\frac{\partial \gamma}{\partial x}(r, X(r), \pi, \theta, z)\right)-\frac{\partial \gamma}{\partial x}(r, X(r), \pi, \theta, z)\right\} \nu(d z) d t \\
& \left.+\int_{t}^{s} \int_{\mathbb{R}_{0}}\left\{\ln \left(1+\frac{\partial \gamma}{\partial x}\left(r, X\left(r^{-}\right), \pi\left(r^{-}, z\right), \theta\left(r^{-}, z\right), z\right)\right)\right\} \widetilde{N}\left(d z, d^{-} r\right)\right] \\
p_{i}(t):= & K_{i}(t)+\int_{t}^{T} \frac{\partial}{\partial x} H_{i}^{0}\left(s, X(s), \pi_{0}(s), \pi_{1}(s, z), \theta_{0}(s), \theta_{1}(s, z)\right) G(t, s) d s \\
q_{i}(t):= & D_{t} p_{i}(t), \\
r_{i}(t, z):= & D_{t, z} p_{i}(t)
\end{aligned}
$$

all exist for $i=1,2,0 \leq t \leq s \leq T, z_{1}, z \in \mathbb{R}_{0}$.

Now let introduce the general Hamiltonians of insiders.

Definition 3.1 The general stochastic Hamiltonians for the stochastic differential game for insiders in Problem 1.1 are the functions

$$
H_{i}(t, x, \pi, \theta, \omega):[0, T] \times \mathbb{R} \times U \times K \times \Omega \longrightarrow \mathbb{R}, i=1,2
$$

defined by

$$
\begin{aligned}
H_{i}(t, x, \pi, \theta, \omega):= & \int_{\mathbb{R}_{0}} f_{i}(t, x, \pi, \theta, z, \omega) \mu(d z)+p_{i}(t)\left(b\left(t, x, \pi_{0}, \theta_{0}, \omega\right)+D_{t+} \sigma\left(t, x, \pi_{0}, \theta_{0}, \omega\right)\right. \\
& \left.+\int_{\mathbb{R}_{0}} D_{t+, z} \gamma(t, x, \pi, \theta, z, \omega) \nu(d z)\right)+q_{i}(t) \sigma\left(t, x, \pi_{0}, \theta_{0}, \omega\right) \\
& +\int_{\mathbb{R}_{0}} r_{i}(t, z)\left\{\gamma(t, x, \pi, \theta, z, \omega)+D_{t+, z} \gamma(t, x, \pi, \theta, z, \omega)\right\} \nu(d z),
\end{aligned}
$$

where $\pi=\left(\pi_{0}, \pi_{1}\right)$ and $\theta=\left(\theta_{0}, \theta_{1}\right)$

We can now state a general stochastic maximum principle of insider for zero-sum games:

Theorem 3.2 [Maximum principle for insider non zero-sum games]

(i) Suppose $(\widehat{\pi}, \widehat{\theta}) \in \mathcal{A}_{\Pi} \times \mathcal{A}_{\Theta}$ is a Nash equilibrium, i.e.

1. $J_{1}(\pi, \widehat{\theta}) \leq J_{1}(\widehat{\pi}, \widehat{\theta})$ for all $\pi \in \mathcal{A}_{\Pi}$

2. $J_{2}(\widehat{\pi}, \theta) \leq J_{2}(\widehat{\pi}, \widehat{\theta})$ for all $\theta \in \mathcal{A}_{\Theta}$ 
Then

$$
\begin{aligned}
& E\left[\left.\nabla_{\pi} \widehat{H}_{1}\left(t, X^{\pi, \widehat{\theta}}(t), \pi, \widehat{\theta}, \omega\right)\right|_{\pi=\widehat{\pi}} \mid \mathcal{G}_{t}^{2}\right]+E[A]=0 \text { a.e. in }(t, \omega), \\
& \text { and } \\
& E\left[\left.\nabla_{\theta} \widehat{H}_{2}\left(t, X^{\widehat{\pi}, \theta}(t), \widehat{\pi}, \theta, \omega\right)\right|_{\theta=\widehat{\theta}} \mid \mathcal{G}_{t}^{1}\right]+E[B]=0 \text { a.e. in }(t, \omega),
\end{aligned}
$$

where $A$ is given by 6.21) and $B$ is defined in a similar way.

$$
\begin{aligned}
\widehat{X}(t)= & X^{(\widehat{\pi}, \widehat{\theta})}(t) \\
\widehat{H}_{i}(t, \widehat{X}(t), \pi, \theta, \omega):= & \int_{\mathbb{R}_{0}} f_{i}(t, \widehat{X}(t), \pi, \theta, z, \omega) \mu(d z) \\
& +\widehat{p}_{i}(t)\left(b\left(t, \widehat{X}(t), \pi_{0}, \theta_{0}, \omega\right)+D_{t+} \sigma\left(t, \widehat{X}(t), \pi_{0}, \theta_{0}, \omega\right)\right. \\
& \left.+\int_{\mathbb{R}_{0}} D_{t+, z} \gamma(t, \widehat{X}(t), \pi, \theta, z, \omega) \nu(d z)\right) \\
& +\widehat{q}_{i}(t) \sigma\left(t, \widehat{X}(t), \pi_{0}, \theta_{0}, \omega\right) \\
& +\int_{\mathbb{R}_{0}} \widehat{r}_{i}(t, z)\left\{\gamma(t, \widehat{X}(t), \pi, \theta, z, \omega)+D_{t+, z} \gamma(t, \widehat{X}(t), \pi, \theta, z, \omega)\right\} \nu(d z),
\end{aligned}
$$

with

$$
\begin{aligned}
\widehat{p}_{i}(t):= & \widehat{K}_{i}(t)+\int_{t}^{T} \frac{\partial}{\partial x} \widehat{H}_{i}^{0}(s, \widehat{X}(s), \widehat{\pi}(s), \widehat{\theta}(s)) \widehat{G}(t, s) d s \\
\widehat{K}_{i}(t):= & g_{i}^{\prime}(\widehat{X}(T))+\int_{t}^{T} \int_{\mathbb{R}_{0}} \frac{\partial}{\partial x} f_{i}(s, \widehat{X}(s), \widehat{\pi}(s, z), \widehat{\theta}(s, z), z) \mu(d z) d s \quad(3.18) \\
\widehat{H}_{i}^{0}(s, \widehat{X}, \widehat{\pi}, \widehat{\theta}):=\widehat{K}_{i}(s)\left(b\left(s, \widehat{X}, \widehat{\pi}_{0}, \widehat{\theta}_{0}\right)+D_{s+} \sigma\left(s, \widehat{X}, \widehat{\pi}_{0}, \widehat{\theta}_{0}\right)\right. & \left.\quad+\int_{\mathbb{R}_{0}} D_{s+, z} \gamma(s, \widehat{X}, \widehat{\pi}, \widehat{\theta}, z) \nu(d z)\right)+D_{s} K_{i}(s) \sigma\left(s, \widehat{X}, \widehat{\pi}_{0}, \widehat{\theta_{0}}\right) \\
& +\int_{\mathbb{R}_{0}} D_{s, z} K_{i}(s)\left\{\gamma(s, \widehat{X}, \widehat{\pi}, \widehat{\theta}, z)+D_{s+, z} \gamma(s, \widehat{X}, \widehat{\pi}, \widehat{\theta}, z)\right\} \nu(d z) \\
\widehat{G}(t, s):= & \exp \left[\int_{t}^{s}\left\{\frac{\partial b}{\partial x}\left(r, \widehat{X}(r), \widehat{\pi}_{0}(r), \widehat{\theta}_{0}(r)\right)-\frac{1}{2}\left(\frac{\partial \sigma}{\partial x}\right)^{2}\left(r, \widehat{X}(r), \widehat{\pi}{ }_{0}(r), \widehat{\theta}_{0}(r)\right)\right\} d r\right. \\
& +\int_{t}^{s} \frac{\partial \sigma}{\partial x}\left(r, \widehat{X}(r), \widehat{\pi}_{0}(r), \widehat{\theta}_{0}(r)\right) d^{-} B(r) \\
& +\int_{t}^{s} \int_{\mathbb{R}_{0}}\left\{\ln \left(1+\frac{\partial \gamma}{\partial x}(r, \widehat{X}(r), \widehat{\pi}, \widehat{\theta}, z)\right)-\frac{\partial \gamma}{\partial x}(r, \widehat{X}(r), \widehat{\pi}, \widehat{\theta}, z)\right\} \nu(d z) d t \\
& \left.+\int_{t}^{s} \int_{\mathbb{R}_{0}}\left\{\ln \left(1+\frac{\partial \gamma}{\partial x}\left(r, \widehat{X}\left(r^{-}\right), \widehat{\pi}\left(r^{-}, z\right), \widehat{\theta}\left(r^{-}, z\right), z\right)\right)\right\} \widehat{N}\left(d z, d^{-} r\right)\right]
\end{aligned}
$$


(ii) Conversely, suppose $(\widehat{\pi}, \widehat{\theta}) \in \mathcal{A}_{\Pi} \times \mathcal{A}_{\Theta}$ such 3.14) and 3.15) hold. Then

$$
\begin{aligned}
& \left.\frac{\partial J_{1}}{\partial y}(\widehat{\pi}+y \beta, \widehat{\theta})\right|_{y=0}=0 \text { for all } \beta \\
& \left.\frac{\partial J_{2}}{\partial v}(\widehat{\pi}, \widehat{\theta}+v \eta)\right|_{v=0}=0 \text { for all } \eta
\end{aligned}
$$

In particular, if

$$
\pi \rightarrow J_{1}(\pi, \widehat{\theta})
$$

and

$$
\theta \rightarrow J_{2}(\widehat{\pi}, \theta)
$$

are concave, then $(\widehat{\pi}, \widehat{\theta})$ is a Nash equilibrium.

Proof. See Appendix.

\section{$3.1 \quad$ Zero-sum games}

Here, we suppose that the given performance functional for Player I is the negative of that for Player II, i.e.,

$$
J_{1}\left(u_{0}, u_{1}\right):=E\left[\int_{0}^{T} f\left(t, X(t), u_{0}(t), u_{1}(t, z), \omega\right) \mu(d z) d t+g(X(T), \omega)\right]=-J_{2}\left(u_{0}, u_{1}\right)
$$

where $E=E_{P}^{x}$ denotes the expectation with respect to $P$ given that $X(0)=x$. Suppose that the controls $u_{0}(t)$ and $u_{1}(t, z)$ have the form $(1.4)$ and $(1.5)$. Let $\mathcal{A}_{\Pi}$ (respectively $\left.\mathcal{A}_{\Theta}\right)$ denote the given family of controls $\pi=\left(\pi_{0}, \pi_{1}\right)$ (respectively $\theta=\left(\theta_{0}, \theta_{1}\right)$ ) such that they are contained in the set of $\mathcal{G}_{t}^{1}$-adapted controls (respectively $\mathcal{G}_{t}^{2}$-adapted controls), 1.1) has a unique strong solution up to time $T$ and

$$
E\left[\int_{0}^{T}\left|f\left(t, X(t), u_{0}(t), u_{1}(t, z), \omega\right)\right| \mu(d z) d t+|g(X(T), \omega)|\right]<\infty .
$$

Then the insider information zero-sum stochastic differential game problem is the following:

Problem 3.3 Find $\pi^{*} \in \mathcal{A}_{\Pi}^{\mathcal{G}^{2}}$ and $\theta^{*} \in \mathcal{A}_{\Theta}^{\mathcal{G}^{1}}$ and $\Phi \in \mathbb{R}$ (if it exists) such that

$$
\Phi=\inf _{\theta \in \mathcal{A}_{\Theta}^{\mathcal{G}^{1}}}\left(\sup _{\pi \in \mathcal{A}_{\Pi}^{\mathcal{G}^{2}}} J(\pi, \theta)\right)=J\left(\pi^{*}, \theta^{*}\right)=\sup _{\pi \in \mathcal{A}_{\Pi}^{\mathcal{G}^{2}}}\left(\inf _{\theta \in \mathcal{A}_{\Theta}^{\mathcal{G}}} J(\pi, \theta)\right)
$$

Such a control $\left(\pi^{*}, \theta^{*}\right)$ is called an optimal control (if it exists). The intuitive idea is that while Player I controls $\pi$, Player II controls $\theta$. The actions of the players are antagonistic, which means that between player I and II there is a payoff $J(\pi, \theta)$ and it is a reward for Player I and cost for Player II. Note that since we allow $b, \sigma, \gamma, f$ and $g$ to be stochastic processes and also because our controls are $\mathcal{G}_{t}^{1}$-adapted, and $\mathcal{G}_{t}^{2}$-adapted respectively, this problem is not of Markovian type and can not be solved by dynamic programming. 
Theorem 3.4 [Maximum principle for insider zero-sum games]

(i) Suppose $(\widehat{\pi}, \widehat{\theta}) \in \mathcal{A}_{\Pi} \times \mathcal{A}_{\Theta}$ is a directional critical point for $J(\pi, \theta)$, in the sense that for all bounded $\beta \in \mathcal{A}_{\Pi}$ and $\eta \in \mathcal{A}_{\Theta}$, there exists $\delta>0$ such that $\widehat{\pi}+y \beta \in \mathcal{A}_{\Pi}, \widehat{\theta}+v \eta \in \mathcal{A}_{\Theta}$ for all $y, v \in(-\delta, \delta)$ and

$$
c(y, v):=J(\widehat{\pi}+y \beta, \widehat{\theta}+v \eta), y, v \in(-\delta, \delta)
$$

has a critical point at zero, i.e.,

$$
\frac{\partial c}{\partial y}(0,0)=\frac{\partial c}{\partial v}(0,0)=0
$$

Then

$$
\begin{aligned}
& E\left[\left.\nabla_{\pi} \widehat{H}\left(t, X^{\pi, \widehat{\theta}}(t), \pi, \widehat{\theta}, \omega\right)\right|_{\pi=\widehat{\pi}} \mid \mathcal{G}_{t}^{2}\right]+E[A]=0 \text { a.e. in }(t, \omega), \\
& \text { and } \\
& E\left[\left.\nabla_{\theta} \widehat{H}\left(t, X^{\widehat{\pi}, \theta}(t), \widehat{\pi}, \theta, \omega\right)\right|_{\theta=\widehat{\theta}} \mid \mathcal{G}_{t}^{1}\right]+E[B]=0 \text { a.e. in }(t, \omega),
\end{aligned}
$$

where $A$ and $B$ are given as in the previous theorem.

$$
\begin{aligned}
\widehat{X}(t)= & X^{(\widehat{\pi}, \widehat{\theta})}(t) \\
\widehat{H}(t, \widehat{X}(t), \pi, \theta, \omega):= & \int_{\mathbb{R}_{0}} f(t, \widehat{X}(t), \pi, \theta, z, \omega) \mu(d z) \\
& +\widehat{p}(t)\left(b\left(t, \widehat{X}(t), \pi_{0}, \theta_{0}, \omega\right)+D_{t+} \sigma\left(t, \widehat{X}(t), \pi_{0}, \theta_{0}, \omega\right)\right. \\
& \left.+\int_{\mathbb{R}_{0}} D_{t+, z} \gamma(t, \widehat{X}(t), \pi, \theta, z, \omega) \nu(d z)\right) \\
& +\widehat{q}(t) \sigma\left(t, \widehat{X}(t), \pi_{0}, \theta_{0}, \omega\right) \\
& +\int_{\mathbb{R}_{0}} \widehat{r}(t, z)\left\{\gamma(t, \widehat{X}(t), \pi, \theta, z, \omega)+D_{t+, z} \gamma(t, \widehat{X}(t), \pi, \theta, z, \omega)\right\} \nu(d z),
\end{aligned}
$$

with

$$
\begin{aligned}
& \widehat{p}(t):=\widehat{K}(t)+\int_{t}^{T} \frac{\partial}{\partial x} \widehat{H}^{0}(s, \widehat{X}(s), \widehat{\pi}(s), \widehat{\theta}(s)) \widehat{G}(t, s) d s \\
& \widehat{K}(t):=g^{\prime}(\widehat{X}(T))+\int_{t}^{T} \int_{\mathbb{R}_{0}} \frac{\partial}{\partial x} f(s, \widehat{X}(s), \widehat{\pi}(s, z), \widehat{\theta}(s, z), z) \mu(d z) d s \\
& \widehat{H}^{0}(s, \widehat{X}, \widehat{\pi}, \widehat{\theta}):=\widehat{K}(s)\left(b\left(s, \widehat{X}, \widehat{\pi}_{0}, \widehat{\theta}_{0}\right)+D_{s+} \sigma\left(s, \widehat{X}, \widehat{\pi}_{0}, \widehat{\theta_{0}}\right)\right. \\
&\left.+\int_{\mathbb{R}_{0}} D_{s+, z} \gamma(s, \widehat{X}, \widehat{\pi}, \widehat{\theta}, z) \nu(d z)\right)+D_{s} K(s) \sigma\left(s, \widehat{X}, \widehat{\pi}_{0}, \widehat{\theta_{0}}\right) \\
&+\int_{\mathbb{R}_{0}} D_{s, z} K(s)\left\{\gamma(s, \widehat{X}, \widehat{\pi}, \widehat{\theta}, z)+D_{s+, z} \gamma(s, \widehat{X}, \widehat{\pi}, \widehat{\theta}, z)\right\} \nu(d z)
\end{aligned}
$$




$$
\begin{aligned}
\widehat{G}(t, s):= & \exp \left[\int_{t}^{s}\left\{\frac{\partial b}{\partial x}\left(r, \widehat{X}(r), \widehat{\pi}_{0}(r), \widehat{\theta}_{0}(r)\right)-\frac{1}{2}\left(\frac{\partial \sigma}{\partial x}\right)^{2}\left(r, \widehat{X}(r), \widehat{\pi}_{0}(r), \widehat{\theta}_{0}(r)\right)\right\} d r\right. \\
& +\int_{t}^{s} \frac{\partial \sigma}{\partial x}\left(r, \widehat{X}(r), \widehat{\pi}_{0}(r), \widehat{\theta}_{0}(r)\right) d B^{-}(r) \\
& +\int_{t}^{s} \int_{\mathbb{R}_{0}}\left\{\ln \left(1+\frac{\partial \gamma}{\partial x}(r, \widehat{X}(r), \widehat{\pi}, \widehat{\theta}, z)\right)-\frac{\partial \gamma}{\partial x}(r, \widehat{X}(r), \widehat{\pi}, \widehat{\theta}, z)\right\} \nu(d z) d t \\
& \left.+\int_{t}^{s} \int_{\mathbb{R}_{0}}\left\{\ln \left(1+\frac{\partial \gamma}{\partial x}\left(r, \widehat{X}\left(r^{-}\right), \widehat{\pi}\left(r^{-}, z\right), \widehat{\theta}\left(r^{-}, z\right), z\right)\right)\right\} \widetilde{N}\left(d z, d^{-} r\right)\right]
\end{aligned}
$$

(ii) Conversely, suppose that there exists a $(\widehat{\pi}, \widehat{\theta}) \in \mathcal{A}_{\Pi} \times \mathcal{A}_{\Theta}$ such that 3.27) and 3.28) hold. Then $(\widehat{\pi}, \widehat{\theta})$ satisfies 3.26 .

\section{Controlled Itô-Lévy processes}

The main result of the previous section (Theorem 3.2) is difficult to apply because of the appearance of the terms $Y(t), D_{t+} Y(t)$ and $D_{t+, z} Y(t)$, which all depend on the control $u$. However, consider the special case when the coefficients do not depend on $X$, i.e., when

$$
\begin{aligned}
& b(t, x, u, \omega)=b(t, u, \omega), \quad \sigma(t, x, u, \omega)=\sigma(t, u, \omega) \\
& \text { and } \theta(t, x, u, z, \omega)=\theta(t, u, z, \omega) .
\end{aligned}
$$

Then equation (1.1) takes the form

$$
\left\{\begin{aligned}
d^{-} X(t)= & b(t, u(t), \omega) d t+\sigma(t, u(t), \omega) d^{-} B(t) \\
& +\int_{\mathbb{R}_{0}} \theta(t, u(t), z, \omega) \widetilde{N}\left(d z, d^{-} t\right) \\
X(0)= & x \in \mathbb{R}
\end{aligned}\right.
$$

We call such processes controlled Itô-Lévy processes.

In this case, Theorem 3.2 simplifies to the following

Theorem 4.1 Let $X(t)$ be a controlled Itô-Lévy process as given in Equation (4.2). Assume that the conditions 15 as in Theorem 3.2 are in force.

Then the following statements are equivalent:

(i) $(\widehat{\pi}, \widehat{\theta})$ is a directional critical point for $J_{i}(\pi, \theta)$ for $i=1,2$ in the sense that for all bounded $\beta \in \mathcal{A}_{\Pi}$ and $\eta \in \mathcal{A}_{\Theta}$, there exists $\delta>0$ such that $\widehat{\pi}+y \beta \in \mathcal{A}_{\Pi}, \widehat{\theta}+v \eta \in \mathcal{A}_{\Theta}$ for all $y, v \in(-\delta, \delta)$.

(ii)

$$
\begin{aligned}
& E\left[L_{\pi}(t) \alpha+M_{\pi}(t) D_{t+} \alpha+\int_{\mathbb{R}_{0}} R_{\pi}(t, z) D_{t+, z} \alpha \nu(d z)\right]=0 \\
& \text { and } \\
& E\left[L_{\theta}(t) \xi+M_{\theta}(t) D_{t+} \xi+\int_{\mathbb{R}_{0}} R_{\theta}(t, z) D_{t+, z} \xi \nu(d z)\right]=0
\end{aligned}
$$


for all $\alpha$ and $\xi$ Malliavin differentiable and all $t \in[0, T]$, where

$$
\begin{aligned}
L_{\pi}(t)= & \widehat{K}_{1}(t)\left(\nabla_{\pi} b(t)+D_{t+} \nabla_{\pi} \sigma(t)+\int_{\mathbb{R}_{0}} D_{t+, z} \nabla_{\pi} \gamma(t, z) \nu(d z)\right) \\
& +\nabla_{\pi} f_{1}(t)+D_{t} \widehat{K}_{1}(t) \nabla_{\pi} \sigma(t) \\
& +\int_{\mathbb{R}_{0}} D_{t, z} \widehat{K}_{1}(t)\left(\nabla_{\pi} \gamma(t, z)+D_{t+, z} \nabla_{\pi} \gamma(t, z)\right) \nu(d z), \\
M_{\pi}(t)= & \widehat{K}_{1}(t) \nabla_{\pi} \sigma(t), \\
R_{\pi}(t, z)= & \left\{\widehat{K}_{1}(t)+D_{t, z} \widehat{K}_{1}(t)\right\}\left(\nabla_{\pi} \gamma(t, z)+D_{t+, z} \nabla_{\pi} \gamma(t, z)\right), \\
L_{\theta}(t)= & \widehat{K}_{2}(t)\left(\nabla_{\theta} b(t)+D_{t+} \nabla_{\theta} \sigma(t)+\int_{\mathbb{R}_{0}} D_{t+, z} \nabla_{\theta} \gamma(t, z) \nu(d z)\right) \\
& +\nabla_{\theta} f_{2}(t)+D_{t} \widehat{K}_{2}(t) \nabla_{\theta} \sigma(t) \\
& +\int_{\mathbb{R}_{0}} D_{t, z} \widehat{K}_{2}(t)\left(\nabla_{\theta} \gamma(t, z)+D_{t+, z} \nabla_{\theta} \gamma(t, z)\right) \nu(d z), \\
M_{\theta}(t)= & \widehat{K}_{2}(t) \nabla_{\theta} \sigma(t) \\
\text { and } & \\
R_{\theta}(t, z)= & \left\{\widehat{K}_{2}(t)+D_{t, z} \widehat{K}_{2}(t)\right\}\left(\nabla_{\theta} \gamma(t, z)+D_{t+, z} \nabla_{\theta} \gamma(t, z)\right) .
\end{aligned}
$$

In particular, if

$$
\pi \rightarrow J_{1}(\pi, \widehat{\theta})
$$

and

$$
\theta \rightarrow J_{2}(\widehat{\pi}, \theta)
$$

are concave, then $(\widehat{\pi}, \widehat{\theta})$ is a Nash equilibrium.

Proof. It is easy to see that in this case, $p(t)=K(t), q(t)=D_{t} K(t), r(t, z)=D_{t, z} K(t)$ and the general Hamiltonian $H_{i}, i=1,2$ given by (3.13) is reduced to $H_{i}$ given as follows

$$
\begin{aligned}
H_{i}(t, x, \pi, \theta, \omega):= & \int_{\mathbb{R}_{0}} f_{i}(t, \pi, \theta, z, \omega) \mu(d z)+p_{i}(t)\left(b\left(t, \pi_{0}, \theta_{0}, \omega\right)+D_{t+} \sigma\left(t, \pi_{0}, \theta_{0}, \omega\right)\right. \\
& \left.+\int_{\mathbb{R}_{0}} D_{t+, z} \gamma(t, \pi, \theta, z, \omega) \nu(d z)\right)+q_{i}(t) \sigma\left(t, \pi_{0}, \theta_{0}, \omega\right) \\
& +\int_{\mathbb{R}_{0}} r_{i}(t, z)\left\{\gamma(t, \pi, \theta, z, \omega)+D_{t+, z} \gamma(t, \pi, \theta, z, \omega)\right\} \nu(d z),
\end{aligned}
$$


(i) Performing the same calculation leads to

$$
\begin{aligned}
A_{1}= & A_{3}=A_{5}=0, \\
A_{2}=E & {\left[\int _ { t } ^ { t + h } \left\{\widehat{K}_{1}(t)\left(\nabla_{\pi} b(s)+D_{s+} \nabla_{\pi} \sigma(s)+\int_{\mathbb{R}_{0}} D_{t s, z} \nabla_{\pi} \gamma(t, z) \nu(d z)\right)\right.\right.} \\
& +D_{t} \widehat{K}_{1}(t) \nabla_{\pi} \sigma(t)+\int_{\mathbb{R}_{0}} \nabla_{\pi} f_{1}(s, z) \mu(d z) \\
& \left.\left.+\int_{\mathbb{R}_{0}} D_{s, z} \widehat{K}_{1}(t)\left(\nabla_{\pi} \gamma(s, z)+D_{s, z} \nabla_{\pi} \gamma(s, z)\right) \nu(d z)\right\} \alpha d s\right], \\
A_{4}=E & {\left[\int_{t}^{t+h} \widehat{K}_{1}(t) \nabla_{\pi} \sigma(s) D_{s+} \alpha d s\right], } \\
A_{6}=E & {\left[\int_{t}^{t+h} \int_{\mathbb{R}_{0}}\left(\widehat{K}_{1}(t)+D_{s, z} \widehat{K}_{1}(t)\right)\left\{\nabla_{\pi} \gamma(s, z)+D_{s+, z} \nabla_{\pi} \gamma(s, z)\right\} \nu(d z) D_{s+, z} \alpha d s\right], }
\end{aligned}
$$

It follows that

$$
\begin{aligned}
&\left.\frac{d}{d h} A_{2}\right|_{h=0}= E\left[\left\{\widehat{K}_{1}(t)\left(\nabla_{\pi} b(t)+D_{t+} \nabla_{\pi} \sigma(t)+\int_{\mathbb{R}_{0}} D_{t+, z} \nabla_{\pi} \gamma(t, z) \nu(d z)\right)\right.\right. \\
&+\nabla_{\pi} f_{1}(t)+D_{t} \widehat{K}_{1}(t) \nabla_{\pi} \sigma(t) \\
&\left.\left.+\int_{\mathbb{R}_{0}} D_{t, z} \widehat{K}_{1}(t)\left(\nabla_{\pi} \gamma(t, z)+D_{t+, z} \nabla_{\pi} \gamma(t, z)\right) \nu(d z)\right\} \alpha\right], \\
&\left.\frac{d}{d h} A_{4}\right|_{h=0}= E\left[\widehat{K}_{1}(t) \nabla_{\pi} \sigma(t) D_{t+} \alpha\right], \\
&\left.\frac{d}{d h} A_{6}\right|_{h=0}=E\left[\int_{\mathbb{R}_{0}}\left\{\widehat{K}_{1}(t)+D_{t, z} \widehat{K}_{1}(t)\right\}\left(\nabla_{\pi} \gamma(t, z)+D_{t+, z} \nabla_{\pi} \gamma(t, z)\right) \nu(d z) D_{t+, z} \alpha\right] .
\end{aligned}
$$

This means that

$$
\begin{aligned}
0= & E\left[\left\{\widehat{K}_{1}(t)\left(\nabla_{\pi} b(t)+D_{t+} \nabla_{\pi} \sigma(t)+\int_{\mathbb{R}_{0}} D_{t+, z} \nabla_{\pi} \gamma(t, z) \nu(d z)\right)\right.\right. \\
& +\nabla_{\pi} f_{1}(t)+D_{t} \widehat{K}_{1}(t) \nabla_{\pi} \sigma(t) \\
& \left.+\int_{\mathbb{R}_{0}} D_{t, z} \widehat{K}_{1}(t)\left(\nabla_{\pi} \gamma(t, z)+D_{t+, z} \nabla_{\pi} \gamma(t, z)\right) \nu(d z)\right\} \alpha \\
& +\widehat{K}_{1}(t) \nabla_{\pi} \sigma(t) D_{t+} \alpha \\
& \left.+\int_{\mathbb{R}_{0}}\left\{\widehat{K}_{1}(t)+D_{t, z} \widehat{K}_{1}(t)\right\}\left(\nabla_{\pi} \gamma(t, z)+D_{t+, z} \nabla_{\pi} \gamma(t, z)\right) \nu(d z) D_{t+, z} \alpha\right] .
\end{aligned}
$$

Performing the same computation for $\mathrm{H}_{2}$, the result follows. This completes the proof for (i).

(ii) The converse part follows from the arguments used in the proof of Theorem 3.2 . 


\subsection{Zero-sum Game}

Under the same hypothesis as given in Section 3.1, if we assume that the controlled process is of Itô-Lévy type, Theorem 3.4 becomes

Theorem 4.2 Let $X(t)$ be a controlled Itô-Lévy process as given in Equation (4.2). Retain the conditions 1 5 as in Theorem 3.2.

Then the following statements are equivalent:

(i) $(\widehat{\pi}, \widehat{\theta})$ is a directional critical point for $J(\pi, \theta)$ in the sense that for all bounded $\beta \in \mathcal{A}_{\Pi}$ and $\eta \in \mathcal{A}_{\Theta}$, there exists $\delta>0$ such that $\widehat{\pi}+y \beta \in \mathcal{A}_{\Pi}, \widehat{\theta}+v \eta \in \mathcal{A}_{\Theta}$ for all $y, v \in(-\delta, \delta)$ and

$$
c(y, v):=J(\widehat{\pi}+y \beta, \widehat{\theta}+v \eta), \quad y, v \in(-\delta, \delta)
$$

has a critical point at 0 , i.e.,

$$
\frac{\partial c}{\partial y}(0,0)=\frac{\partial c}{\partial v}(0,0)=0
$$

(ii)

$$
\begin{aligned}
& E\left[L_{\pi}(t) \alpha+M_{\pi}(t) D_{t+} \alpha+\int_{\mathbb{R}_{0}} R_{\pi}(t, z) D_{t+, z} \alpha \nu(d z)\right]=0 \\
& \text { and } \\
& E\left[L_{\theta}(t) \xi+M_{\theta}(t) D_{t+} \xi+\int_{\mathbb{R}_{0}} R_{\theta}(t, z) D_{t+, z} \xi \nu(d z)\right]=0
\end{aligned}
$$

for all $\alpha$ and $\xi$ Malliavin differentiable and all $t \in[0, T]$, where

$$
\begin{aligned}
L_{\pi}(t)= & \widehat{K}(t)\left(\nabla_{\pi} b(t)+D_{t+} \nabla_{\pi} \sigma(t)+\int_{\mathbb{R}_{0}} D_{t+, z} \nabla_{\pi} \gamma(t, z) \nu(d z)\right) \\
& +\nabla_{\pi} f(t)+D_{t} \widehat{K}(t) \nabla_{\pi} \sigma(t) \\
& +\int_{\mathbb{R}_{0}} D_{t, z} \widehat{K}(t)\left(\nabla_{\pi} \gamma(t, z)+D_{t+, z} \nabla_{\pi} \gamma(t, z)\right) \nu(d z), \\
M_{\pi}(t)= & \widehat{K}(t) \nabla_{\pi} \sigma(t), \\
R_{\pi}(t, z)= & \left\{\widehat{K}(t)+D_{t, z} \widehat{K}(t)\right\}\left(\nabla_{\pi} \gamma(t, z)+D_{t+, z} \nabla_{\pi} \gamma(t, z)\right), \\
L_{\theta}(t)= & \widehat{K}(t)\left(\nabla_{\theta} b(t)+D_{t+} \nabla_{\theta} \sigma(t)+\int_{\mathbb{R}_{0}} D_{t+, z} \nabla_{\theta} \gamma(t, z) \nu(d z)\right) \\
& +\nabla_{\theta} f(t)+D_{t} \widehat{K}(t) \nabla_{\theta} \sigma(t) \\
& +\int_{\mathbb{R}_{0}} D_{t, z} \widehat{K}(t)\left(\nabla_{\theta} \gamma(t, z)+D_{t+, z} \nabla_{\theta} \gamma(t, z)\right) \nu(d z), \\
M_{\theta}(t)= & \widehat{K}(t) \nabla_{\theta} \sigma(t) \\
\text { and } & \\
R_{\theta}(t, z)= & \left\{\widehat{K}(t)+D_{t, z} \widehat{K}(t)\right\}\left(\nabla_{\theta} \gamma(t, z)+D_{t+, z} \nabla_{\theta} \gamma(t, z)\right) .
\end{aligned}
$$




\subsection{Some special cases revisited}

The results obtained so far are for given general sup-filtrations. To provide some concrete examples let us confine ourselves to particular cases of filtrations which are first chaos generated (see [19]). This can arise when

- the insider always has information in advance compared to the honest trader. This means that if $\mathcal{G}_{t}$ and $\mathcal{F}_{t}$ represent the information flow of the insider and the honest respectively, then we have $\mathcal{G}_{t} \supset \mathcal{F}_{t+\delta(t)}$ where $\delta(t)>0$;

- the trader has from the very beginning a particular information about the future (initial enlargement of filtration). This means that if $\mathcal{G}_{t}$ and $\mathcal{F}_{t}$ represent the information flow of the insider and the honest, then $\mathcal{G}_{t}=\mathcal{F}_{t} \vee \sigma\left(L_{1}\right) \vee \cdots \vee \sigma\left(L_{n}\right)$ where $L_{i}, \quad i=1, \cdots, n$ are e.g. Brownian integrals of deterministic functions.

Let $\mathcal{B}$ be one of the following sup-filtrations,

$$
\begin{aligned}
& \mathcal{B}_{1}=\mathcal{F}_{t+\delta(t)}, \\
& \mathcal{B}_{2}=\mathcal{F}_{t} \vee \sigma\left(B_{T}\right), \\
& \mathcal{B}_{3}=\mathcal{F}_{[0, t] \cup O},
\end{aligned}
$$

where $O$ is an open set contained in $[0, T]$. Then it can be shown (see [19]) that $\mathcal{B}_{i}, i=$ $1, \cdots, 3$ are the first chaos generated $\sigma$-algebras.

From now on we assume that the following conditions are fulfilled:

Fix a $t_{0} \in[0, T]$. Then

(C1) There exist a $\mathcal{A}^{i}=\mathcal{A}_{t_{0}}^{i} \subseteq \mathbb{D}_{1,2} \cap L^{2}\left(\mathcal{G}_{t_{0}}^{i}\right), i=1,2$ and a measurable $\mathcal{M}^{i} \subset\left[t_{0}, T\right], i=$ 1,2 such that $D_{t} \alpha$ and $D_{t, z} \alpha$ are $\mathcal{G}_{t_{0}}^{i}$-measurable, for all $\alpha \in \mathcal{A}^{i}, \quad t \in \mathcal{M}^{i}, i=1,2$,

(C2) $D_{t+} \alpha=D_{t} \alpha$ and $D_{t+, z} \alpha=D_{t, z} \alpha$ for all $\alpha \in \mathcal{A}^{i}$ and a.a. $t, z, t \in \mathcal{M}^{i}, i=1,2$.

(C3) $\mathcal{A}^{i}$ is total in $L^{2}\left(\mathcal{G}_{t_{0}}^{i}\right), \quad i=1,2$,

(C4) $E\left[M_{\theta}(t) \mid \mathcal{G}_{t_{0}}^{1}\right] \cdot \chi_{[0, t] \cap \mathcal{M}^{1}}, E\left[R_{\theta}(t, z) \mid \mathcal{G}_{t_{0}}^{1}\right] \cdot \chi_{[0, t] \cap \mathcal{M}^{1}}, E\left[M_{\pi}(t) \mid \mathcal{G}_{t_{0}}^{2}\right] \cdot \chi_{[0, t] \cap \mathcal{M}^{2}}$ and $\left.E\left[R_{\pi}(t, z)\right) \mid \mathcal{G}_{t_{0}}^{2}\right] \cdot$ $\chi_{[0, t] \cap \mathcal{M}^{2}}$ are Skorohod integrable for all $t$,

(C5) $\int_{0}^{T}\left\{\left|E\left[L_{\theta}(t) \mid \mathcal{G}_{t_{0}}^{1}\right]\right|+\left|E\left[L_{\pi}(t) \mid \mathcal{G}_{t_{0}}^{1}\right]\right|\right\} d t<\infty \quad$ a.e.,

where $L_{\pi}, M_{\pi}, L_{\theta}, M_{\theta}, R_{\pi}$ and $R_{\theta}$ are defined as in (4.3), (4.4), (4.6), (4.7), (4.5) and 4.8).

Remark 4.3 In [10], a filtration satisfying (C1)-(C3) is called smoothly anticipative filtration.

Theorem 4.4 Suppose that $\mathcal{G}^{i}, i=1,2$ satisfied (C1)-(C5). Suppose that $(\widehat{\pi}, \widehat{\theta})$ is a directional critical point for $J_{i}(\pi, \theta)$ for $i=1,2$ in the sense that for all bounded $\beta \in \mathcal{A}_{\Pi}$ and 
$\eta \in \mathcal{A}_{\Theta}$, there exists a $\delta>0$ such that $\widehat{\pi}+y \beta \in \mathcal{A}_{\Pi}, \widehat{\theta}+v \eta \in \mathcal{A}_{\Theta}$ for all $y, v \in(-\delta, \delta)$. Then for all $h_{i}=\chi_{\left[t_{0}, s\right)}(t) \chi_{\mathcal{M}^{i}}(t), \quad i=1,2$

$$
\begin{aligned}
0= & E\left[\int_{0}^{T} E\left[L_{\theta_{i}}(t) \mid \mathcal{H}^{i}\right] h_{i}(t) d t+\int_{0}^{T} E\left[M_{\theta_{i}}(t) \mid \mathcal{H}^{i}\right] h_{i}(t) \delta B_{t}\right. \\
& \left.+\int_{0}^{T} \int_{\mathbb{R}_{0}} E\left[R_{\theta_{i}}(t, z) \mid \mathcal{H}^{i}\right] h_{i}(t) \tilde{N}(\delta t, d z) \mid \mathcal{H}^{i}\right] .
\end{aligned}
$$

where $\mathcal{H}^{i}=G_{t_{0}}^{i}, i=1,2, \theta_{1}=\theta, \theta_{2}=\pi$.

Proof. See Theorem 5.7 in [10].

Corollary 4.5 Assume that the conditions in Theorem 4.4 are in force. In addition, we require that $E\left[M_{\theta_{i}}(t) \mid \mathcal{H}^{i}\right] \in \mathbb{M}_{1,2}^{B}, i=1,2$. Set $X^{i}(t)=E\left[B(t) \mid \mathcal{H}^{i}\right]$. Then

$$
\begin{aligned}
0= & \int_{0}^{T} E\left[L_{\theta_{i}}(t) \mid \mathcal{H}^{i}\right] h_{i}(t) d t+\int_{0}^{T} E\left[M_{\theta_{i}}(t) \mid \mathcal{H}^{i}\right] h_{i}(t) d^{-} X^{i}(t) \\
& -\int_{0}^{T} D_{t^{+}} E\left[M_{\theta_{i}}(t) \mid \mathcal{H}^{i}\right] h_{i}(t) d t
\end{aligned}
$$

where $\theta_{i}, h_{i}$, and $\mathcal{H}^{i}$ are as in Theorem 4.4.

Proof. Note that $X^{i}(t), i=1,2$ has continuous version and has existing quadratic variation (since $B(t)$ has quadratic variation). By Lemma 2.3 and by assumption, we know that

$$
\int_{0}^{T} E\left[M_{\theta_{i}}(t) \mid \mathcal{H}^{i}\right] h_{i}(t) \delta B_{t}=\int_{0}^{T} E\left[M_{\theta_{i}}(t) \mid \mathcal{H}^{i}\right] h_{i}(t) d^{-} B(t)-\int_{0}^{T} D_{t^{+}} E\left[M_{\theta_{i}}(t) \mid \mathcal{H}^{i}\right] h_{i}(t) d t
$$

It follows from condition (C1) that

$$
\begin{aligned}
E\left[\int_{0}^{T} E\left[M_{\theta_{i}}(t) \mid \mathcal{H}^{i}\right] h_{i}(t) \delta B_{t} \mid \mathcal{H}^{i}\right]= & E\left[\int_{0}^{T} E\left[M_{\theta_{i}}(t) \mid \mathcal{H}^{i}\right] h_{i}(t) d^{-} B(t) \mid \mathcal{H}^{i}\right] \\
& -\int_{0}^{T} D_{t} E\left[M_{\theta_{i}}(t) \mid \mathcal{H}^{i}\right] h_{i}(t) d t .
\end{aligned}
$$

On the other hand, using uniform convergence on compacts (ucp) in $L^{1}(P)$, we observe that

$$
\begin{aligned}
E\left[\int_{0}^{T} E\left[M_{\theta_{i}}(t) \mid \mathcal{H}^{i}\right] h_{i}(t) d^{-} B(t) \mid \mathcal{H}^{i}\right] & =E\left[\lim _{\epsilon \rightarrow 0^{+}} \int_{0}^{T} E\left[M_{\theta_{i}}(t) \mid \mathcal{H}^{i}\right] h_{i}(t) \frac{B(t+\epsilon)-B(t)}{\epsilon} d t \mid \mathcal{H}^{i}\right] \\
& =\lim _{\epsilon \rightarrow 0^{+}} E\left[\int_{0}^{T} E\left[M_{\theta_{i}}(t) \mid \mathcal{H}^{i}\right] h_{i}(t) \frac{B(t+\epsilon)-B(t)}{\epsilon} d t \mid \mathcal{H}^{i}\right] \\
& =\lim _{\epsilon \rightarrow 0^{+}} \int_{0}^{T} E\left[M_{\theta_{i}}(t) \mid \mathcal{H}^{i}\right] h_{i}(t) E\left[\frac{B(t+\epsilon)-B(t)}{\epsilon} \mid \mathcal{H}^{i}\right] d t \\
& =\lim _{\epsilon \rightarrow 0^{+}} \int_{0}^{T} E\left[M_{\theta_{i}}(t) \mid \mathcal{H}^{i}\right] h_{i}(t) \frac{X^{i}(t+\epsilon)-X^{i}(t)}{\epsilon} d t \\
& =\int_{0}^{T} E\left[M_{\theta_{i}}(t) \mid \mathcal{H}^{i}\right] h_{i}(t) d^{-} X^{i}(t)(\text { in the ucp sense) }
\end{aligned}
$$


From the previous arguments, we can deduce the following results

Theorem 4.6 [Brownian case] Assume that the conditions in Corollary 4.5 are satisfied. Suppose that $b$ and $\sigma$ do not depend on the controlled process $X(\cdot)$. Set $X_{t}^{i}:=E\left[B(t) \mid \mathcal{G}_{t_{0}}^{i}\right], \quad i=$ 1,2 . Let the quadratic variation $\left[X^{i}\right]$ of $X^{i}, i=1,2$ be non-zero. The the following statement are equivalent

(i) $(\widehat{\pi}, \widehat{\theta})$ is a directional critical point for $J_{i}(\pi, \theta)$ for $i=1,2$ in the sense that for all bounded $\beta \in \mathcal{A}_{\Pi}$ and $\eta \in \mathcal{A}_{\Theta}$, there exists a $\delta>0$ such that $\widehat{\pi}+y \beta \in \mathcal{A}_{\Pi}, \widehat{\theta}+v \eta \in \mathcal{A}_{\Theta}$ for all $y, v \in(-\delta, \delta)$.

(ii)

(1) $E\left[L_{\pi}(t) \mid \mathcal{G}_{t_{0}}^{2}\right]=E\left[M_{\pi}(t) \mid \mathcal{G}_{t_{0}}^{2}\right]=0$, dt - a.e on $\left(\operatorname{supp} d\left[X^{2}\right]\right) \cap\left(t_{0}, T\right], P-$ a.e

(2) $E\left[L_{\theta}(t) \mid \mathcal{G}_{t_{0}}^{1}\right]=E\left[M_{\theta}(t) \mid \mathcal{G}_{t_{0}}^{1}\right]=0$, dt-a.e on $\left(\operatorname{supp} d\left[X^{1}\right]\right) \cap\left(t_{0}, T\right], P-$ a.e

where $L_{\pi}, M_{\pi}, L_{\theta}$, and $M_{\theta}$ are given by (4.3), (4.4), (4.6) and (4.7), respectively and where $\operatorname{supp} d\left[X^{i}\right]$ denotes the support of the measure induced by the quadratic variation of the process $X^{i}, i=1,2$. In particular, if

$$
\pi \rightarrow J_{1}(\pi, \widehat{\theta})
$$

and

$$
\theta \rightarrow J_{2}(\widehat{\pi}, \theta)
$$

are concave, then $(\widehat{\pi}, \widehat{\theta})$ is a Nash-equilibrium.

Proof. Note that

$$
\left[\int_{0} E\left[M_{\theta_{i}}(t) \mid \mathcal{H}^{i}\right] h_{i}(t) d^{-} X^{i}(t)\right]_{T}=\int_{0}^{T}\left(E\left[M_{\theta_{i}}(t) \mid \mathcal{H}^{i}\right] h_{i}(t)\right)^{2} d\left[X^{i}\right]_{t} .
$$

We conclude from 4.17 that

$$
\int_{0}^{T}\left(E\left[M_{\theta_{i}}(t) \mid \mathcal{H}^{i}\right] h_{i}(t)\right)^{2} d\left[X^{i}\right]_{t}=0 P-\text { a.e. }
$$

The proof follows.

Remark 4.7 If $\left[X^{i}\right], i=1,2$ are non-zero, then $\left(B(t), \mathcal{G}_{t}^{i}\right), i=1,2$ cannot belong to the class of Dirichlet processes. Examples which satisfy $(\boldsymbol{C 1})-(\boldsymbol{C} 3)$ (for $\mathcal{M} \in\left(t_{0}, T\right]$ ) are $\mathcal{B}_{1}$ or $\mathcal{B}_{3}$. See also Theorem 4.8.

Theorem 4.8 [Mixed case] Suppose that $b, \sigma$ and $\theta$ do not depend on $X(\cdot)$ and that

$$
\mathcal{G}^{i}=\mathcal{B}_{1} \text { or } \mathcal{G}^{i}=\mathcal{B}_{3}, i=1,2 .
$$

In addition assume that $(\boldsymbol{C} 4)-(\boldsymbol{C 5})$ are valid for $\mathcal{M} \in\left(t_{0}, T\right]$. Then the following statements are equivalent: 
(i) $(\widehat{\pi}, \widehat{\theta})$ is a directional critical point for $J_{i}(\pi, \theta)$ for $i=1,2$ in the sense that for all bounded $\beta \in \mathcal{A}_{\Pi}$ and $\eta \in \mathcal{A}_{\Theta}$, there exists a $\delta>0$ such that $\widehat{\pi}+y \beta \in \mathcal{A}_{\Pi}, \widehat{\theta}+v \eta \in \mathcal{A}_{\Theta}$ for all $y, v \in(-\delta, \delta)$.

(ii)

$$
\begin{aligned}
(\text { iii }) E\left[L_{\pi}(t) \mid \mathcal{G}_{t_{0}}^{2}\right] & =E\left[M_{\pi}(t) \mid \mathcal{G}_{t_{0}}^{2}\right]=E\left[R_{\pi}(t, z) \mid \mathcal{G}_{t_{0}}^{2}\right]=0, \\
(\text { iv }) E\left[L_{\theta}(t) \mid \mathcal{G}_{t_{0}}^{1}\right] & =E\left[M_{\theta}(t) \mid \mathcal{G}_{t_{0}}^{1}\right]=E\left[R_{\theta}(t, z) \mid \mathcal{G}_{t_{0}}^{1}\right]=0,
\end{aligned}
$$

where $L_{\pi}, M_{\pi}, R_{\pi}, L_{\theta}, M_{\theta}$ and $R_{\theta}$ are given by (4.3), (4.4), (4.5), (4.6), (4.7) and (4.8) respectively. In particular, if

$$
\pi \rightarrow J_{1}(\pi, \widehat{\theta})
$$

and

$$
\theta \rightarrow J_{2}(\widehat{\pi}, \theta)
$$

are concave, then $(\widehat{\pi}, \widehat{\theta})$ is a Nash-equilibrium.

In order to study the case of the initial enlargement of filtration, we need the following Theorem which is based on Theorem 5.8 in [10]:

Theorem 4.9 [Brownian case] Adopting the notation of Section 5.1 in [10], suppose that $\mathcal{G}^{i}, i=1,2$ satisfied $(\boldsymbol{C 1})-(\boldsymbol{C} 3)$ and $\gamma=0$. Suppose that $(\widehat{\pi}, \widehat{\theta})$ is a directional critical point for $J_{i}(\pi, \theta)$ for $i=1,2$ in the sense that for all bounded $\beta \in \mathcal{A}_{\Pi}$ and $\eta \in \mathcal{A}_{\Theta}$, there exists a $\delta>0$ such that $\widehat{\pi}+y \beta \in \mathcal{A}_{\Pi}, \widehat{\theta}+v \eta \in \mathcal{A}_{\Theta}$ for all $y, v \in(-\delta, \delta)$. In addition, we required that $E\left[M_{\theta_{i}}(t) \mid \mathcal{G}_{t^{-}}^{i}\right] \in \mathbb{M}_{1,2}^{B}$ and are forward integrable with respect to $E\left[d^{-} B(t) \mid \mathcal{G}_{t^{-}}^{i}\right]$. Then

$$
\begin{aligned}
0= & \int_{0}^{T} E\left[L_{\theta_{i}}(t) \mid \mathcal{G}_{t^{-}}^{i}\right] h_{0}(t) d t+\int_{0}^{T} E\left[M_{\theta_{i}}(t) \mid \mathcal{G}_{t^{-}}^{i}\right] h_{0}(t) E\left[d^{-} B(t) \mid \mathcal{G}_{t^{-}}^{i}\right] \\
& -\int_{0}^{T} D_{t^{+}} E\left[M_{\theta_{i}}(t) \mid \mathcal{G}_{t^{-}}^{i}\right] h_{0}(t) d t
\end{aligned}
$$

for all bounded deterministic functions $h_{0}(t)$, where $\theta_{1}=\theta, \theta_{2}=\pi$.

Proof. See Theorem 5.8 in [10].

It follows from the preceding Theorem and Theorem 5.11 in [10] that

Theorem 4.10 [Brownian case] Suppose that $\mathcal{G}^{i}=\mathcal{F}_{t} \vee \sigma(B(T)), i=1,2$. Suppose the conditions of Theorem 4.9 are satisfied. Assume that $(\widehat{\pi}, \widehat{\theta})$ is a directional critical point for $J_{i}(\pi, \theta)$ for $i=1,2$ in the sense that for all bounded $\beta \in \mathcal{A}_{\Pi}$ and $\eta \in \mathcal{A}_{\Theta}$, there exists a $\delta>0$ such that $\widehat{\pi}+y \beta \in \mathcal{A}_{\Pi}, \widehat{\theta}+v \eta \in \mathcal{A}_{\Theta}$ for all $y, v \in(-\delta, \delta)$. Then

$$
E\left[L_{\theta_{i}}(t) \mid \mathcal{G}_{t^{-}}^{i}\right]+E\left[M_{\theta_{i}}(t) \mid \mathcal{G}_{t^{-}}^{i}\right] \frac{B(T)-B(t)}{T-t}=0, \text { for a.a. } t \in[0, T] .
$$

where $\theta_{1}=\theta, \theta_{2}=\pi$.

In the next section, we apply our results to model a competition of two heterogeneously informed agents in the market. We particularly focus on a game between the market and the trader. We assume that the mean relative growth rate $\theta(t)$ of the risky asset is not known to the trader, but subject to uncertainty. 


\section{Application to optimal and competing-insider trading}

Consider a financial market with two investments possibilities:

1. A risk free asset, where the unit price $S_{0}(t)$ at time $t$ is given by

$$
d S_{0}(t)=r(t) S_{0}(t) d t, \quad S_{0}(0)=1 .
$$

2. A risky asset, where the unit price $S_{1}(t)$ at time $t$ is given by the stochastic differential equation

$$
d S_{1}(t)=S_{1}\left(t^{-}\right)\left[\theta(t) d t+\sigma_{0}(t) d^{-} B(t)+\int_{\mathbb{R}_{0}} \gamma(t, z) \tilde{N}\left(d^{-} t, d z\right)\right], \quad S_{1}(0)>0 .
$$

Here $r(t) \geq 0, \theta(t), \sigma_{0}(t)$, and $\gamma(t, z) \geq-1+\epsilon$ (for some constant $\epsilon>0$ ) are given $\mathcal{G}_{t}^{1-}$ predictable, forward integrable processes, where $\left\{\mathcal{G}_{t}^{1}\right\}_{t \in[0, T]}$ is a given filtration such that

$$
\mathcal{F}_{t} \subset \mathcal{G}_{t}^{1} \text { for all } t \in[0, T]
$$

Suppose a trader in this market is an insider, in the sense that she has access to information represented by $\mathcal{G}_{t}^{2}$ at time $t$ (with $\mathcal{F}_{t} \subset \mathcal{G}_{t}^{2}$ for all $t \in[0, T]$ ). Assume that $\mathcal{G}_{t}^{1} \subset \mathcal{G}_{t}^{2}$ (e.g. $\left.\mathcal{G}_{t}^{1}=\mathcal{F}_{t}\right)$. Let $\pi(t)=\pi(t, \omega)$ be a portfolio representing the amount invested by her in the risky asset at time $t$. Then this portfolio is a $\mathcal{G}_{t}^{2}$-predictable stochastic process and hence the corresponding wealth process $X(t)=X^{(\pi, \theta)}(t)$ will then satisfy the (forward) SDE

$$
\begin{aligned}
d^{-} X(t)= & \frac{X(t)-\pi(t)}{S_{0}(t)} d S_{0}(t)+\frac{\pi(t)}{S_{1}(t)} d^{-} S_{1}(t) \\
= & X(t) r(t) d t+\pi(t)\left[(\theta(t)-r(t)) d t+\sigma_{0}(t) d^{-} B(t)\right. \\
& \left.+\int_{\mathbb{R}_{0}} \gamma(t, z) \widetilde{N}\left(d^{-} t, d z\right)\right], \quad t \in[0, T] \\
X(0)= & x>0
\end{aligned}
$$

By choosing $S_{0}(t)$ as a numeraire, we can, without loss of generality, assume that

$$
r(t)=0
$$

from now on. Then Equations (5.4) and (5.5) simplify to

$$
\begin{cases}d^{-} X(t) & =\pi(t)\left[\theta(t) d t+\sigma_{0}(t) d^{-} B(t)+\int_{\mathbb{R}_{0}} \gamma(t, z) \tilde{N}\left(d^{-} t, d z\right)\right] \\ X(0) & =x>0\end{cases}
$$

This is a controlled Itô-Lévy process of the type discussed in Section 4 . Let us assume that the mean relative growth rate $\theta(t)$ of the risky asset is not known to the trader, but subject to uncertainty. We may regard $\theta$ as a market scenario or a stochastic control of the market, which is playing against the trader. Let $\mathcal{A}_{\Pi}^{\mathcal{G}^{2}}$ and $\mathcal{A}_{\Theta}^{\mathcal{G}^{1}}$ denote the set of admissible controls 
$\pi, \theta$, respectively. The worst case insider information scenario optimal problem for the trader is to find $\pi^{*} \in \mathcal{A}_{\Pi}^{\mathcal{G}^{2}}$ and $\theta^{*} \in \mathcal{A}_{\Theta}^{\mathcal{G}^{1}}$ and $\Phi \in \mathbb{R}$ such that

$$
\begin{aligned}
\Phi & =\inf _{\theta \in \mathcal{A}_{\Theta}^{\mathcal{G}^{1}}}\left(\sup _{\pi \in \mathcal{A}_{\Pi}^{\mathcal{G}^{2}}} E\left[U\left(X^{\theta, \pi}\right)(T)\right]\right) \\
& =E\left[U\left(X^{\theta^{*}, \pi^{*}}\right)(T)\right]
\end{aligned}
$$

where $U: \mathbb{R}_{+} \rightarrow \mathbb{R}$ is a given utility function, assumed to be concave, strictly increasing and $C^{1}$. We want to study this problem by using results of Section 4 . In this case, the processes $K(t), L(t), M(t)$ and $R(t, z)$ which are given respectively by equations (3.7), (4.3), (4.4), (4.5), (4.6), 4.7) and 4.8 become

$$
\begin{aligned}
K_{1}(t)= & K_{2}(t)=U^{\prime}(X(T)) \\
L_{\pi}(t)= & U^{\prime}(X(T))\left[\theta(t)+D_{t+} \sigma_{0}(t)+\int_{\mathbb{R}_{0}} D_{t+, z} \gamma(t, z) \nu(d z)\right] \\
& +\int_{\mathbb{R}_{0}} D_{t, z} U^{\prime}(X(T))\left[\gamma(t, z)+D_{t+, z} \gamma(t, z)\right] \nu(d z)+D_{t} U^{\prime}(X(T)) \sigma_{0}(t) \\
M_{\pi}(t)= & U^{\prime}(X(T)) \sigma_{0}(t) \\
R_{\pi}(t, z)= & \left\{U^{\prime}(X(T))+D_{t, z} U^{\prime}(X(T))\right\}\left\{\gamma(t, z)+D_{t+, z} \gamma(t, z)\right\} \\
L_{\theta}(t)= & U^{\prime}(X(T)) \pi \\
M_{\theta}(t)= & R_{\theta}(t, z)=0
\end{aligned}
$$

Therefore Theorem 4.6 and Theorem 4.8 of Section 4 imply the following:

Theorem 5.1 Suppose that $\sigma_{0}(t) \neq 0$ and that either

$$
\mathcal{G}^{i}=\mathcal{B}_{1} \text { or } \mathcal{B}_{3}, i=1,2 . \quad \text { (Mixed case) }
$$

or the quadratic variation of $X^{i}(t)=E\left[B(t) \mid \mathcal{G}_{t_{0}}^{i}\right]$ is non-zero, $i=1,2$, (Brownian case).

Then there does not exist an optimal solution $\left(\pi^{*}, \theta^{*}\right) \in \mathcal{A}_{\Pi}^{\mathcal{G}^{2}} \times \mathcal{A}_{\Theta}^{\mathcal{G}^{1}}$ of the stochastic differential game (5.8).

Proof. Suppose an optimal portfolio exists. Without loss of generality we only consider the first case. Then we have seen that

$$
E\left[L_{\pi}(t) \mid \mathcal{G}_{t_{0}}^{2}\right]=E\left[M_{\pi}(t) \mid \mathcal{G}_{t_{0}}^{2}\right]=E\left[R_{\pi}(t, z) \mid \mathcal{G}_{t}^{2}\right]=E\left[L_{\theta}(t) \mid \mathcal{G}_{t_{0}}^{1}\right]=0
$$

for a.a. $t \in\left(t_{0}, T\right], z \in \mathbb{R}_{0}$. In particular,

$$
\begin{aligned}
& E\left[M_{\pi}(t) \mid \mathcal{G}_{t_{0}}^{2}\right]=E\left[U^{\prime}(X(T)) \mid \mathcal{G}_{t_{0}}^{2}\right] \sigma_{0}(t)=0, \\
& \text { or } \\
& E\left[L_{\theta}(t) \mid \mathcal{G}_{t_{0}}^{1}\right]=E\left[U^{\prime}(X(T)) \mid \mathcal{G}_{t_{0}}^{1}\right] \pi(t)=0 .
\end{aligned}
$$

Choosing $t=T$ and let $t_{0} \uparrow T$, we get $U^{\prime}(X(T))=0$, which contradicts our assumption about $U$. Hence an optimal portfolio cannot exist. 
Remark 5.2 The previous result is in accordance with Theorem 1 in [11], since the Brownian motion is not a semimartingale neither in the filtration $\mathcal{B}_{1}$ nor in the filtration $\mathcal{B}_{3}$.

Theorem 5.3 (Knowing the terminal value of the risky asset) Suppose that $\sigma_{0}(t) \neq 0 \mathcal{G}_{t}^{1}=$ $\mathcal{F}_{t}$ and $\mathcal{G}_{t}^{2}=\mathcal{F}_{t} \vee \sigma\left(S_{1}(T)\right), t \in[0, T]$ and the coefficients $\theta(t), \sigma_{0}(t)=\sigma_{0} \neq 0$ and $\gamma(t, z) \equiv 0$ are deterministic. Further, require that the conditions $\left(\boldsymbol{C H}_{4}\right)-(\boldsymbol{C 5})$ hold for $\mathcal{M} \in\left(t_{0}, T\right]$ and that

1. $E\left[M_{\theta}(t) \mid \mathcal{G}_{t_{0}}^{1}\right], E\left[M_{\pi}(t) \mid \mathcal{G}_{t_{0}}^{2}\right] \in \mathbb{M}_{1,2}^{B}$

2. $\underset{t \uparrow T}{\lim } E\left[\left|D_{t^{+}} E\left[M_{\phi_{i}}(t) \mid \mathcal{G}_{t_{0}}^{2}\right]\right|\right]<\infty$ for $\phi_{1}=\theta$ and $\phi_{2}=\pi$.

3. $\frac{\lim }{t \uparrow T} E\left[\left|L_{\phi_{i}}(t)\right|\right]<\infty$ for $\phi_{1}=\theta$ and $\phi_{2}=\pi$.

Then, there does not exist an optimal portfolio for the insider.

Proof. Since $S_{1}(t)$ can be written as

$$
S_{1}(t)=S_{1}(0) \exp \left(\int_{0}^{T}\left\{\theta(t)-\frac{1}{2} \sigma_{0}^{2}(t)\right\} d t+\int_{0}^{T} \sigma_{0}(t) d B(t)\right),
$$

One finds that $\mathcal{G}_{t}^{2}=\mathcal{B}_{t}^{2}$. Hence the result follows from Theorem 6.3 in [10].

Remark 5.4 It can be shown (see [10]) that Theorem 5.3 also applies e.g to cases, when the terminal value $S_{1}(T)$ is given by $\max _{o \leq t \leq T} B(t)$ or $\eta(T)$, where $\eta$ is a Lévy process.

\section{Application to optimal insider consumption}

Suppose we have a cash flow $X(t)=X^{(\pi, \theta)}(t)$ given by

$$
\left\{\begin{array}{l}
d X(t)=(\theta(t)-u(t)) d t+\sigma(t) d B(t)+\int_{\mathbb{R}_{0}} \gamma(t, z) \tilde{N}(d t, d z), \\
X(0)=x \in \mathbb{R} .
\end{array}\right.
$$

Here $\theta(t), \sigma(t)$ and $\theta(t, z)$ are given $\mathcal{F}_{T}$-measurable processes and $\pi(t) \geq 0$ is the consumption rate, assumed to be adapted to a given insider filtration $\left\{\mathcal{G}_{t}\right\}_{t \in[0, T]}$ where

$$
\mathcal{F}_{t} \subset \mathcal{G}_{t} \text { for all } t
$$

Let $f(t, \pi, \theta, \omega) ; t \in[0, T], \pi, \theta \in \mathbb{R}, \omega \in \Omega$ be a given $\mathcal{F}_{T}$-measurable utility process. Assume that $u \rightarrow f(t, \pi, \theta, \omega)$ is strictly increasing, concave and $C^{1}$ for a.a $(t, \omega)$.

Let $g(x, \omega) ; x \in \mathbb{R}, \omega \in \Omega$ be a given $\mathcal{F}_{T}$-measurable random variable for each $x$. Assume that $u \rightarrow g(x, \omega)$ is concave for a.a $\omega$. Define the performance functional $J$ by

$$
J(\pi, \theta)=E\left[\int_{0}^{T} f(t, \pi(t), \theta(t), \omega) d t+g\left(X^{(u)}(T), \omega\right)\right] ; u \in \mathcal{A}_{\mathcal{G}}, u \geq 0
$$

Note that $\pi \rightarrow J(\pi, \widehat{\theta})$ and $\theta \rightarrow J(\widehat{\pi}, \theta)$ are concave, so $(\widehat{\pi}, \widehat{\theta})$ is a Nash-equilibrium if and only if $(\widehat{\pi}, \widehat{\theta})$ is a critical point of $J(\pi, \theta)$. 
Theorem 6.1 [Optimal insider consumption stochastic differential game consumption I] $(\widehat{\pi}, \widehat{\theta})$ is a Nash-equilibrium of insider consumption rate for the performance functional $J$ in Equation (6.2) if and only if

$$
-E\left[\frac{\partial}{\partial \theta} f(t, \widehat{\pi}(t), \widehat{\theta}(t), \omega) \mid \mathcal{G}_{t}\right]=E\left[\frac{\partial}{\partial \pi} f(t, \widehat{\pi}(t), \widehat{\theta}(t), \omega) \mid \mathcal{G}_{t}\right]=E\left[g^{\prime}\left(X^{(\widehat{\pi}, \widehat{\theta})}(T), \omega\right) \mid \mathcal{G}_{t}\right]
$$

Proof. In this case we have

$$
\begin{aligned}
K_{1}(t) & =K_{2}(t)=g\left(X^{(\pi, \theta)}(T)\right) \\
L_{\pi}(t) & =-g\left(X^{(\pi, \theta)}(T)\right)+\frac{\partial}{\partial \pi} f(t, \widehat{\pi}(t), \widehat{\theta}(t)) \\
L_{\theta}(t) & =g\left(X^{(\pi, \theta)}(T)\right)+\frac{\partial}{\partial \theta} f(t, \widehat{\pi}(t), \widehat{\theta}(t)) \\
M_{\pi}(t) & =R_{\pi}(t, z)=M_{\theta}=R_{\theta}=0
\end{aligned}
$$

Therefore $(\widehat{\pi}, \widehat{\theta})$ is a critical point for $J(\pi, \theta)$ if and only if

$$
\begin{aligned}
0 & =E\left[L_{\pi}(t) \mid \mathcal{G}_{t}\right]=E\left[L_{\theta}(t) \mid \mathcal{G}_{t}\right] \\
& =E\left[\frac{\partial}{\partial \pi} f(t, \widehat{\pi}(t), \widehat{\theta}(t)) \mid \mathcal{G}_{t}\right]+E\left[-g^{\prime}\left(X^{(\widehat{\pi}, \widehat{\theta}(t))}(T)\right) \mid \mathcal{G}_{t}\right] \\
& =E\left[\frac{\partial}{\partial \theta} f(t, \widehat{\pi}(t), \widehat{\theta}(t)) \mid \mathcal{G}_{t}\right]+E\left[g^{\prime}\left(X^{(\widehat{\pi}, \widehat{\theta}(t))}(T)\right) \mid \mathcal{G}_{t}\right]
\end{aligned}
$$

Since $X^{(\widehat{\pi}, \widehat{\theta})}(T)$ depends on $(\widehat{\pi}, \widehat{\theta})$, Equation 6.3 does not give the value of $\widehat{\pi}(t)$ (respectively $\widehat{\theta}(t))$ directly.

However, in some special cases $\widehat{\pi}$ and $\widehat{\theta}(t)$ can be found explicitly:

Corollary 6.2 (Optimal insider stochastic differential game consumption II) Assume that

$$
g(x, \omega)=\lambda(\omega) x
$$

for some $\mathcal{F}_{T}$-measurable random variable $\lambda \geq 0$.

Then the Nash-equilibrium $(\widehat{\pi}(t), \widehat{\theta}(t))$ of the stochastic differential game (6.2) is given by

$$
\begin{aligned}
E\left[\frac{\partial}{\partial \pi} f(t, \widehat{\pi}, \widehat{\theta}, \omega) \mid \mathcal{G}_{t}\right]_{\pi=\widehat{\pi}(t)} & =E\left[\lambda \mid \mathcal{G}_{t}\right] \\
E\left[\frac{\partial}{\partial \theta} f(t, \widehat{\pi}, \widehat{\theta}, \omega) \mid \mathcal{G}_{t}\right]_{\theta=\widehat{\theta}(t)} & =-E\left[\lambda \mid \mathcal{G}_{t}\right]
\end{aligned}
$$

Thus we see that the Nash-equilibrium exists, for any given insider information filtration $\left\{\mathcal{G}_{t}\right\}_{t \geq 0}$. 


\section{References}

[1] An, T. T. K., Øksendal, B.: A maximum principle for stochastic differential games with partial information. J. of Optim. Theory and Appl. 139, p. 463483 (2008).

[2] An, T. T. K., Øksendal, B., Okur, Y. Y.: A Malliavin calculus approach to general stochastic differential games with partial information . Preprint (2008).

[3] Baghery, F., Øksendal, B.: A maximum principle for stochastic control with partial information. Stochastic Analysis and Applications 25, p. 705-717 (2007).

[4] Bertoin, J.: Lévy processes. Volume 121 of Cambridge Tracts in Mathematics. Cambridge University Press, Cambridge, (1996).

[5] Biagini, F., Øksendal, B.: A general stochastic calculus approach to insider trading. Applied Mathematics and Optimization 52, p. 167-181 (2005).

[6] Di Nunno, G., Meyer-Brandis, T., Øksendal, B., Proske, F.: Malliavin calculus and anticipative Itô formulae for Lévy processes. Inf. dim. Anal. Quant. Probab. 8, p. 235258 (2005).

[7] Di Nunno, G., Meyer-Brandis, T., Øksendal, B., Proske, F.: Optimal portfolio for an insider in a market driven by Lévy processes. Quant. Fin. 6 (1) p. 83-94 (2006).

[8] Di Nunno, G., Øksendal, B., Proske, F.: Malliavin Calculus for Lévy Processes with Applications to Finance. Universitext Springer, (2008).

[9] Di Nunno, G., Øksendal, B., Pamen, M. O., Proske, F.: Uniqueness of Decompositions of Skorohod-Semimartingales. http://www.math.uio.no/eprint/pure_math/2009/1009.pdf. Submitted (2009).

[10] Di Nunno, G., Øksendal, B., Pamen, M. O., Proske, F.: A general maximum principle for anticipative stochastic control and applications to insider trading. Submitted (2009).

[11] Ewald, C-O., Xiao Y.: Information: Price and impact on general welfare and optimal investment. An anticipating stochastic differential game model. Preprint (2007).

[12] Framstad, N., Øksendal, B., Sulem, A.: Stochastic maximum principle for optimal control of jump diffusions and applications to finance. J. Optimization Theory and Appl. 121 (1), p. 77-98 (2004). Errata: J.Optimization Theory and Appl. 124 (2), p. 511-512 (2005).

[13] Ghomrasni, R. and Pamen, O. M.: An approximation of the generalized covariation process. Submitted (2008).

[14] Karatzas, I., Pikovsky, I.: Anticipating portfolio optimization. Adv. Appl. Probab. 28: 1095-1122 (1996).

[15] Kohatsu-Higa, A., Sulem, A.: Utility Maximization in an Insider Influenced Market. Mathematical Finance. 16 (1), p. 153-179 (2006). 
[16] Meyer-Brandis, T., Øksendal, B., Zhou, X.: A Malliavin calculus approach to a general maximum principle for stochastic control. Submitted. Eprint, University of Oslo 10/2008.

[17] Nualart, D.: The Malliavin Calculus and Related Topics. Springer (1995).

[18] Nualart, D., Pardoux, E.: Stochastic calculus with anticipating integrands. Probability Theory and Related Fields 78, 535-581 (1988).

[19] Nualart, D., Üstünel, A.S., Zakai, E.: Some Relations among Classes of $\sigma$-fields on Wiener Space. Probability Theory and Related Fields 85, 119-119 (1990).

[20] Øksendal, B., Sulem, A.: Applied Stochastic Control of Jump Diffusions. SpringerVerlag, Berlin, second edition, (2007).

[21] Russo, F., Vallois, P.: Forward, backward and symmetric stochastic integration. Probability Theory and Related Fields 97, p. 403-421 (1993).

[22] Russo, F., Vallois, P.: Stochastic calculus with respect to continuous finite variation processes. Stochastics and Stochastics Reports 70, 1-40 (2000).

[23] Sato, Ken-iti.: Lévy Processes and Infinitely Divisible Distributions. Volume 68 of Cambridge Studies in Advanced Mathematics.Cambridge University Press, Cambridge, (1999). 


\section{Appendix: Proof of Theorem 3.2}

Proof. The proof relies on a combination of arguments of [2] and [10].

(i) Suppose $(\widehat{\pi}, \widehat{\theta}) \in \mathcal{A}_{\Pi} \times \mathcal{A}_{\Theta}$ is a Nash equilibrium. Since 1 and 2 hold for all $\pi$ and $\theta$, ( $\left.\widehat{\pi}, \widehat{\theta}\right)$ is a directional critical point for $J_{i}(\pi, \theta)$ for $i=1,2$ in the sense that for all bounded $\beta \in \mathcal{A}_{\Pi}$ and $\eta \in \mathcal{A}_{\Theta}$, there exists $\delta>0$ such that $\widehat{\pi}+y \beta \in \mathcal{A}_{\Pi}, \widehat{\theta}+v \eta \in \mathcal{A}_{\Theta}$ for all $y, v \in(-\delta, \delta)$. Then we have

$$
\begin{aligned}
0= & \left.\frac{\partial}{\partial y} J_{1}(\widehat{\pi}+y \beta, \widehat{\theta})\right|_{y=0} \\
= & E^{x}\left[\int _ { 0 } ^ { T } \int _ { \mathbb { R } _ { 0 } } \left\{\left.\frac{\partial}{\partial x} f_{1}\left(t, \widehat{X}(t), \widehat{\pi}_{0}(t), \widehat{\pi}_{1}(t, z), \widehat{\theta}_{0}(t), \widehat{\theta}_{1}(t, z), z\right) \frac{d}{d y} X^{(\widehat{\pi}+y \beta, \theta)}(t)\right|_{y=0}\right.\right. \\
& \left.+\left.\nabla_{\pi} f_{1}\left(t, X^{(\pi, \widehat{\theta})}(t), \pi_{0}(t), \pi_{1}(t, z), \widehat{\theta}_{0}(t), \widehat{\theta}_{1}(t, z), z\right)\right|_{\widehat{\pi}=\pi} \beta^{*}(t)\right\} \mu(d z) d t \\
& \left.+\left.g^{\prime}(X(T)) \frac{d}{d y} X^{(\widehat{\pi}+y \beta, \theta)}(t)\right|_{y=0}\right] \\
= & E^{x}\left[\int _ { 0 } ^ { T } \int _ { \mathbb { R } _ { 0 } } \left\{\frac{\partial}{\partial x} f_{1}\left(t, \widehat{X}(t), \widehat{\pi}_{0}(t), \widehat{\pi}_{1}(t, z), \widehat{\theta}_{0}(t), \widehat{\theta}_{1}(t, z), z\right) \widehat{Y}(t)\right.\right. \\
& \left.+\left.\nabla_{\pi} f_{1}\left(t, X^{(\pi, \widehat{\theta})}(t), \pi_{0}(t), \pi_{1}(t, z), \widehat{\theta}_{0}(t), \widehat{\theta}_{1}(t, z), z\right)\right|_{\widehat{\pi}=\pi} \beta^{*}(t)\right\} \mu(d z) d t \\
& \left.+g^{\prime}(\widehat{X}(T)) \widehat{Y}(t)\right]
\end{aligned}
$$

Where

$$
\begin{aligned}
\widehat{Y}(t)= & \widehat{Y}_{\beta}(t)=\left.\frac{d}{d y} X^{(\widehat{\pi}+y \beta, \widehat{\theta})}(t)\right|_{y=0} \\
= & \int_{0}^{t}\left\{\frac{\partial}{\partial x} b\left(s, \widehat{X}(s), \widehat{\pi}_{0}(s), \widehat{\theta}_{0}(s)\right) Y(s)\right. \\
& \left.+\left.\nabla_{\pi} b\left(s, X^{\pi, \widehat{\theta}}(s), \pi_{0}(s), \widehat{\theta}_{0}(s)\right)\right|_{\pi=\widehat{\pi}} \beta^{*}(s)\right\} d s \\
& +\int_{0}^{t}\left\{\frac{\partial}{\partial x} \sigma\left(s, \widehat{X}(s), \widehat{\pi}_{0}(s), \widehat{\theta}_{0}(s)\right) Y(s)\right. \\
& \left.+\left.\nabla_{\pi} \sigma\left(s, X^{\pi, \widehat{\theta}}(s), \pi_{0}(s), \widehat{\theta}_{0}(s)\right)\right|_{\pi=\widehat{\pi}} \beta^{*}(s)\right\} d B^{-}(s) \\
& +\int_{0}^{t} \int_{\mathbb{R}_{0}}\left\{\frac{\partial}{\partial x} \gamma\left(s, \widehat{X}\left(s^{-}\right), \widehat{\pi}_{0}\left(s^{-}\right), \widehat{\theta}_{0}\left(s^{-}\right), z\right) Y(s)\right. \\
& \left.+\left.\nabla_{\pi} \gamma\left(s, X^{\pi, \widehat{\theta}}\left(s^{-}\right), \pi_{0}\left(s^{-}\right), \widehat{\theta}_{0}\left(s^{-}\right), z\right)\right|_{\pi=\widehat{\pi}} \beta^{*}(s)\right\} \widetilde{N}\left(d z, d^{-} s\right)
\end{aligned}
$$

We study the three summands separately. Using the short notation $\frac{\partial}{\partial x} f_{1}(t, \widehat{X}(t), \widehat{\pi}, \widehat{\theta}, z)=$ $\frac{\partial}{\partial x} f_{1}(t, z),\left.\nabla_{\pi} f_{1}\left(t, X^{(\pi, \widehat{\theta})}(t), \pi, \widehat{\theta}, z\right)\right|_{\widehat{\pi}=\pi}$ and similarly for $\frac{\partial}{\partial x} b, \nabla_{\pi} b, \frac{\partial}{\partial x} \sigma, \nabla_{\pi} \sigma, \frac{\partial}{\partial x} \gamma$ and $\nabla_{\pi} \gamma$ 
By the duality formulas (2.5) and $(2.8)$ and the Fubini theorem, we get

$$
\begin{aligned}
E & {\left[g_{1}^{\prime}(X(T)) Y(T)\right] } \\
=E & {\left[g _ { 1 } ^ { \prime } ( X ( T ) ) \left(\int_{0}^{T}\left\{\frac{\partial b}{\partial x}(t) Y(t)+\nabla_{\pi} b(t) \beta^{*}(t)\right\} d t\right.\right.} \\
& +\int_{0}^{T}\left\{\frac{\partial \sigma}{\partial x}(t) Y(t)+\nabla_{\pi} \sigma(s) \beta^{*}(t)\right\} d^{-} B(t) \\
& \left.\left.+\int_{0}^{T} \int_{\mathbb{R}_{0}}\left\{\frac{\partial \gamma}{\partial x}\left(t, z_{1}\right) Y(t)+\nabla_{\pi} \gamma\left(s, z_{1}\right) \beta^{*}(t)\right\} \tilde{N}\left(d z_{1}, d^{-} t\right)\right)\right] \\
=E & {\left[\int_{0}^{T} g_{1}^{\prime}(X(T))\left\{\frac{\partial b}{\partial x}(t) Y(t)+\nabla_{\pi} b(t) \beta^{*}(t)\right\} d t\right] } \\
& +E\left[\int_{0}^{T} D_{t} g_{1}^{\prime}(X(T))\left\{\frac{\partial \sigma}{\partial x}\left(t, z_{1}\right) Y(t)+\nabla_{\pi} \sigma\left(t, z_{1}\right) \beta^{*}(t)\right\} d t\right] \\
& +E\left[\int_{0}^{T} g_{1}^{\prime}(X(T)) D_{t+}\left(\frac{\partial \sigma}{\partial x}(t) Y(t)+\nabla_{\pi} \sigma(t) \beta^{*}(t)\right) d t\right] \\
& +E\left[\int_{0}^{T} \int_{\mathbb{R}_{0}} D_{t, z_{1}} g_{1}^{\prime}(X(T))\left\{\frac{\partial \gamma}{\partial x}\left(t, z_{1}\right) Y(t)+\nabla_{\pi} \gamma\left(t, z_{1}\right) \beta^{*}(t)\right\} \nu\left(d z_{1}\right) d t\right] \\
& +E\left[\int _ { 0 } ^ { T } \int _ { \mathbb { R } _ { 0 } } \{ g _ { 1 } ^ { \prime } ( X ( T ) ) + D _ { t , z _ { 1 } } g _ { 1 } ^ { \prime } ( X ( T ) ) \} D _ { t + , z _ { 1 } } \left(\frac{\partial \gamma}{\partial x}\left(t, z_{1}\right) Y(t)\right.\right. \\
& \left.\left.+\nabla_{\pi} \gamma\left(t, z_{1}\right) \beta^{*}(t)\right) \nu\left(d z_{1}\right) d t\right]
\end{aligned}
$$

Changing notation $z_{1} \rightarrow z$ this becomes

$$
\begin{aligned}
= & E\left[\int _ { 0 } ^ { T } \left\{g_{1}^{\prime}(X(T))\left(\frac{\partial b}{\partial x}(t)+D_{t+} \frac{\partial \sigma}{\partial x}(t)+\int_{\mathbb{R}_{0}} D_{t+, z} \frac{\partial \gamma}{\partial x}(t, z) \nu(d z)\right)\right.\right. \\
& \left.\left.+D_{t} g_{1}^{\prime}(X(T)) \frac{\partial \sigma}{\partial x}(t)+\int_{\mathbb{R}_{0}} D_{t, z} g_{1}^{\prime}(X(T))\left(\frac{\partial \gamma}{\partial x}(t, z)+D_{t+, z} \frac{\partial \gamma}{\partial x}(t, z)\right) \nu(d z)\right\} Y(t) d t\right] \\
& +E\left[\int _ { 0 } ^ { T } \left\{g_{1}^{\prime}(X(T))\left(\nabla_{\pi} b(t)+D_{t+} \nabla_{\pi} \sigma(t)+\int_{\mathbb{R}_{0}} D_{t+, z} \nabla_{\pi} \gamma(t, z) \nu(d z)\right)\right.\right. \\
& \left.\left.+D_{t} g_{1}^{\prime}(X(T)) \nabla_{\pi} \sigma(t)+\int_{\mathbb{R}_{0}} D_{t, z} g_{1}^{\prime}(X(T))\left(\nabla_{\pi} \gamma(t, z)+D_{t+, z} \nabla_{\pi} \gamma(t, z)\right) \nu(d z)\right\} \beta^{*}(t) d t\right] \\
& +E\left[\int_{0}^{T} g_{1}^{\prime}(X(T)) \frac{\partial \sigma}{\partial x}(t) D_{t+} Y(t) d t\right] \\
+ & E\left[\int_{0}^{T} g_{1}^{\prime}(X(T)) \nabla_{\pi} \sigma(t) D_{t+} \beta^{*}(t) d t\right] \\
+ & E\left[\int_{0}^{T} \int_{\mathbb{R}_{0}}\left\{g_{1}^{\prime}(X(T))+D_{t, z} g_{1}^{\prime}(X(T))\right\}\left\{\frac{\partial \gamma}{\partial x}(t, z)+D_{t+, z} \frac{\partial \gamma}{\partial x}(t, z)\right\} D_{t+, z} Y(t) \nu(d z) d t\right] \\
& +E\left[\int_{0}^{T} \int_{\mathbb{R}_{0}}\left\{g_{1}^{\prime}(X(T))+D_{t, z} g_{1}^{\prime}(X(T))\right\}\left\{\nabla_{\pi} \gamma(t, z)+D_{t+, z} \nabla_{\pi} \gamma(t, z)\right\} D_{t+, z} \beta^{*}(t) \nu(d z) d t\right] .
\end{aligned}
$$


Here we used the multidimensional product rule for Malliavin derivatives.

Similarly, we have using both Fubini and duality formulas (2.5) and (2.8), we get

$$
\begin{aligned}
& E^{x}\left[\int_{0}^{T} \int_{\mathbb{R}_{0}} \frac{\partial f_{1}}{\partial x}(t, z) Y(t) \mu(d z) d t\right] \\
= & E^{x}\left[\int _ { 0 } ^ { T } \int _ { \mathbb { R } _ { 0 } } \frac { \partial f _ { 1 } } { \partial x } ( t , z ) \left(\int_{0}^{t}\left\{\frac{\partial b}{\partial x}(s) Y(s)+\nabla_{\pi} b(s) \beta^{*}(s)\right\} d s\right.\right. \\
& +\int_{0}^{t}\left\{\frac{\partial \sigma}{\partial x}(s) Y(s)+\nabla_{\pi} \sigma(s) \beta^{*}(s)\right\} d^{-} B(s) \\
& \left.\left.+\int_{0}^{t} \int_{\mathbb{R}_{0}}\left\{\frac{\partial \gamma}{\partial x}\left(s, z_{1}\right) Y(s)+\nabla_{\pi} \gamma\left(s, z_{1}\right)\right\} \tilde{N}\left(d z_{1}, d^{-} s\right)\right) d t\right] \\
= & E^{x}\left[\int_{0}^{T} \int_{\mathbb{R}_{0}}\left(\int_{0}^{t} \frac{\partial f_{1}}{\partial x}(t, z)\left\{\frac{\partial b}{\partial x}(s) Y(s)+\nabla_{\pi} b(s) \beta^{*}(s)\right\} d s\right) \mu(d z) d t\right] \\
& +E^{x}\left[\int_{0}^{T} \int_{\mathbb{R}_{0}}\left(\int_{0}^{t} D_{s} \frac{\partial f_{1}}{\partial x}(t, z)\left\{\frac{\partial \sigma}{\partial x}(s) Y(s)+\nabla_{\pi} \sigma(s) \beta^{*}(s)\right\} d s\right) \mu(d z) d t\right] \\
& +E\left[\int_{0}^{T} \int_{\mathbb{R}_{0}}\left(\int_{0}^{t} \frac{\partial f_{1}}{\partial x}(t, z) D_{s+}\left\{\frac{\partial \sigma(s)}{\partial x} Y(s)+\nabla_{\pi} \sigma(s) \beta^{*}(s)\right\} d s\right) \mu(d z) d t\right] \\
& +E\left[\int_{0}^{T} \int_{\mathbb{R}_{0}}\left(\int_{0}^{t} \int_{\mathbb{R}_{0}} D_{s, z_{1}} \frac{\partial f_{1}}{\partial x}(t, z)\left\{\frac{\partial \gamma}{\partial x}\left(s, z_{1}\right) Y(s)+\nabla_{\pi} \gamma\left(s, z_{1}\right) \beta^{*}(s)\right\} \nu\left(d z_{1}\right) d s\right) \mu(d z) d t\right] \\
& +E\left[\int _ { 0 } ^ { T } \int _ { \mathbb { R } _ { 0 } } \left(\int_{0}^{t} \int_{\mathbb{R}_{0}}\left\{\frac{\partial f_{1}}{\partial x}(t, z)+D_{s, z_{1}} \frac{\partial f_{1}}{\partial x}(t, z)\right\} \times\right.\right. \\
& \left.\left.D_{s+, z_{1}}\left(\frac{\partial \gamma}{\partial x}\left(s, z_{1}\right) Y(s)+\nabla_{\pi} \gamma\left(s, z_{1}\right) \beta(s)\right) \nu\left(d z_{1}\right) d s\right) \mu(d z) d t\right] .
\end{aligned}
$$


Changing notation $t_{1} \rightarrow t$ and $z_{1} \rightarrow z$ this becomes

$$
\begin{aligned}
& =E^{x}\left[\int _ { 0 } ^ { T } \left\{\left(\int_{t}^{T} \int_{\mathbb{R}_{0}} \frac{\partial f_{1}}{\partial x}(s, z) \mu(d z) d s\right)\left(\frac{\partial b}{\partial x}(t)+D_{t+} \frac{\partial \sigma}{\partial x}(t)+\int_{\mathbb{R}_{0}} D_{t+, z} \frac{\partial \gamma}{\partial x}(t, z) \nu(d z)\right)\right.\right. \\
& +\left(\int_{t}^{T} \int_{\mathbb{R}_{0}} D_{t} \frac{\partial f_{1}}{\partial x}(s, z) \mu(d z) d s\right) \frac{\partial \sigma}{\partial x}(t) \\
& \left.\left.+\int_{\mathbb{R}_{0}}\left(\int_{t}^{T} \int_{\mathbb{R}_{0}} D_{t, z} \frac{\partial f_{1}}{\partial x}(s, z) \mu(d z) d s\right)\left(\frac{\partial \gamma}{\partial x}(t, z)+D_{t+, z} \frac{\partial \gamma}{\partial x}(t, z)\right) \nu(d z)\right\} Y(t) d t\right] \\
& +E^{x}\left[\int _ { 0 } ^ { T } \left\{\left(\int_{t}^{T} \int_{\mathbb{R}_{0}} \frac{\partial f}{\partial x}(s, z) \mu(d z) d s\right)\left(\nabla_{\pi} b(t)+D_{t+} \nabla_{\pi} \sigma(t)+\int_{\mathbb{R}_{0}} D_{t+, z} \nabla_{\pi} \gamma(t, z) \nu(d z)\right)\right.\right. \\
& +\left(\int_{t}^{T} \int_{\mathbb{R}_{0}} D_{t} \frac{\partial f_{1}}{\partial x}(s, z) \mu(d z) d s\right) \nabla_{\pi} \sigma(t) \\
& \left.\left.+\left(\int_{t}^{T} \int_{\mathbb{R}_{0}} D_{t, z} \frac{\partial f_{1}}{\partial x}(s, z) \mu(d z) d s\right)\left(\nabla_{\pi} \gamma(t, z)+D_{t+, z} \nabla_{\pi} \gamma(t, z)\right) \nu(d z)\right\} \beta^{*}(t) d t\right] \\
& +E^{x}\left[\int_{0}^{T}\left(\int_{t}^{T} \int_{\mathbb{R}_{0}} \frac{\partial f_{1}}{\partial x}(s, z) \mu(d z) d s\right) \frac{\partial \sigma(t)}{\partial x} D_{t+} Y(t) d t\right] \\
& +E^{x}\left[\int_{0}^{T}\left(\int_{t}^{T} \int_{\mathbb{R}_{0}} \frac{\partial f_{1}}{\partial x}(s, z) \mu(d z) d s\right) \nabla_{\pi} \sigma(t) D_{t+} \beta^{*}(t) d t\right] \\
& +E^{x}\left[\int_{0}^{T} \int_{\mathbb{R}_{0}}\left\{\int_{t}^{T} \int_{\mathbb{R}_{0}}\left(\frac{\partial f}{\partial x}(s, z)+D_{t, z} \frac{\partial f}{\partial x}(s, z)\right) \mu(d z) d s\right\} \times\right. \\
& \left.\left\{\frac{\partial \gamma}{\partial x}(t)+D_{t+, z} \frac{\partial \gamma}{\partial x}(t)\right\} D_{t+, z} Y(t) \nu(d z) d t\right] \\
& +E^{x}\left[\int_{0}^{T} \int_{\mathbb{R}_{0}}\left\{\int_{t}^{T} \int_{\mathbb{R}_{0}}\left(\frac{\partial f_{1}}{\partial x}(s, z)+D_{t, z} \frac{\partial f_{1}}{\partial x}(s, z)\right) \mu(d z) d s\right\} \times\right. \\
& \left.\left\{\nabla_{\pi} \gamma(t, z)+D_{t+, z} \nabla_{\pi} \gamma(t, z)\right\} D_{t+, z} \beta^{*}(t) \nu(d z) d t\right] .
\end{aligned}
$$

Recall that

$$
K(t):=g_{1}^{\prime}(X(T))+\int_{t}^{T} \int_{\mathbb{R}_{0}} \frac{\partial f_{1}}{\partial x}\left(s, z_{1}\right) \mu\left(d z_{1}\right) d s
$$

so

$$
\widehat{K}_{1}(t):=g_{1}^{\prime}(\widehat{X}(T))+\int_{t}^{T} \int_{\mathbb{R}_{0}} \frac{\partial f_{1}}{\partial x}\left(s, z_{1}\right) \mu\left(d z_{1}\right) d s .
$$


By combining (6.9)- 6.10$)$, we get

$$
\begin{aligned}
0= & E\left[\int _ { 0 } ^ { T } \left\{\widehat{K}_{1}\left(\frac{\partial b}{\partial x}(t)+D_{t+} \frac{\partial \sigma}{\partial x}(t)+\int_{\mathbb{R}_{0}} D_{t+, z} \frac{\partial \gamma}{\partial x}(t, z) \nu(d z)\right)\right.\right. \\
& \left.\left.+D_{t} \widehat{K}_{1} \frac{\partial \sigma}{\partial x}(t)+\int_{\mathbb{R}_{0}} D_{t, z} \widehat{K}_{1}\left(\frac{\partial \gamma}{\partial x}(t, z)+D_{t+, z} \frac{\partial \gamma}{\partial x}(t, z)\right) \nu(d z)\right\} Y(t) d t\right] \\
& +E\left[\int _ { 0 } ^ { T } \left\{\widehat{K}_{1}\left(\nabla_{\pi} b(t)+D_{t+} \nabla_{\pi} \sigma(t)+\int_{\mathbb{R}_{0}} D_{t+, z} \nabla_{\pi} \gamma(t, z) \nu(d z)\right)\right.\right. \\
& \left.\left.+D_{t} \widehat{K}_{1} \nabla_{\pi} \sigma(t)+\int_{\mathbb{R}_{0}} D_{t, z} \widehat{K}_{1}\left(\nabla_{\pi} \gamma(t, z)+D_{t+, z} \nabla_{\pi} \gamma(t, z)\right) \nu(d z)\right\} \beta^{*}(t) d t\right] \\
& +E\left[\int_{0}^{T} \widehat{K}_{1} \frac{\partial \sigma(t)}{\partial x} D_{t+} Y(t) d t\right]+E\left[\int_{0}^{T} \widehat{K}_{1} \nabla_{\pi} \sigma(t) D_{t+} \beta^{*}(t) d t\right] \\
& +E\left[\int_{0}^{T} \int_{\mathbb{R}_{0}}\left(\widehat{K}_{1}+D_{t, z} \widehat{K}_{1}\right)\left\{\frac{\partial \gamma}{\partial x}(t)+D_{t+, z} \frac{\partial \gamma}{\partial x}(t)\right\} D_{t+, z} Y(t) \nu(d z) d t\right] \\
& +E\left[\int_{0}^{T} \int_{\mathbb{R}_{0}}\left(\widehat{K}_{1}+D_{t, z} \widehat{K}_{1}\right)\left\{\nabla_{\pi} \gamma(t, z)+D_{t+, z} \nabla_{\pi} \gamma(t, z)\right\} D_{t+, z} \beta^{*}(t) \nu(d z) d t\right] \\
& +E\left[\int_{0}^{T} \int_{\mathbb{R}_{0}} \nabla_{\pi} f_{1}(t, z) \beta^{*}(t) \mu(d z) d t\right] .
\end{aligned}
$$

Now apply this to $\beta=\beta_{\alpha} \in \mathcal{A}_{\Pi}$ given as $\beta_{\alpha}(s):=\alpha \chi_{[t, t+h]}(s)$, for some $t, h \in(0, T), t+$ $h \leq T$, where $\alpha=\alpha(\omega)$ is bounded and $\mathcal{G}_{t}^{2}$-measurable. Then $Y^{\left(\beta_{\alpha}\right)}(s)=0$ for $0 \leq s \leq t$ and hence (6.12) becomes

$$
A_{1}+A_{2}+A_{3}+A_{4}+A_{5}+A_{6}=0
$$

Where

$$
\begin{aligned}
A_{1}= & E^{x}\left[\int _ { t } ^ { T } \left\{\widehat{K}_{1}(t)\left(\frac{\partial b}{\partial x}(s)+D_{s+} \frac{\partial \sigma}{\partial x}(s)+\int_{\mathbb{R}_{0}} D_{s+, z} \frac{\partial \gamma}{\partial x}(s, z) \nu(d z)\right)+D_{t} \widehat{K}_{1}(t) \frac{\partial \sigma}{\partial x}(t)\right.\right. \\
& \left.\left.+\int_{\mathbb{R}_{0}} D_{s, z} \widehat{K}_{1}(t)\left(\frac{\partial \gamma}{\partial x}(s, z)+D_{s+, z} \frac{\partial \gamma}{\partial x}(s, z)\right) \nu(d z)\right\} Y^{\left(\beta_{\alpha}\right)}(s) d s\right], \\
A_{2}= & E^{x}\left[\int _ { t } ^ { t + h } \left\{\widehat{K}_{1}(t)\left(\nabla_{\pi} b(s)+D_{s+} \nabla_{\pi} \sigma(s)+\int_{\mathbb{R}_{0}} D_{t s, z} \nabla_{\pi} \gamma(t, z) \nu(d z)\right)+D_{t} \widehat{K}_{1}(t) \nabla_{\pi} \sigma(t)\right.\right. \\
& \left.\left.+\int_{\mathbb{R}_{0}} D_{s, z} \widehat{K}_{1}(t)\left(\nabla_{\pi} \gamma(s, z)+D_{s, z} \nabla_{\pi} \gamma(s, z)\right) \nu(d z)+\int_{\mathbb{R}_{0}} \nabla_{\pi} f_{1}(s, z) \mu(d z)\right\} \alpha d s\right], \\
A_{3}= & E^{x}\left[\int_{t}^{T} \widehat{K}_{1}(t) \frac{\partial \sigma(s)}{\partial x} D_{s+} Y^{\left(\beta_{\alpha}\right)}(s) d s\right], \\
A_{4}=+ & E\left[\int_{t}^{t+h} \widehat{K}_{1}(t) \nabla_{\pi} \sigma(s) D_{s+} \alpha d s\right], \\
A_{5}= & E^{x}\left[\int_{t}^{T} \int_{\mathbb{R}_{0}}\left(\widehat{K}_{1}(t)+D_{s, z} \widehat{K}_{1}(t)\right)\left\{\frac{\partial \gamma}{\partial x}(s)+D_{s+, z} \frac{\partial \gamma}{\partial x}(t)\right\} \nu(d z) D_{s+, z} Y^{\left(\beta_{\alpha}\right)}(s) d s\right], \\
A_{6}= & E^{x}\left[\int_{t}^{t+h} \int_{\mathbb{R}_{0}}\left(\widehat{K}_{1}(t)+D_{s, z} \widehat{K}_{1}(t)\right)\left\{\nabla_{\pi} \gamma(s, z)+D_{s+, z} \nabla_{\pi} \gamma(s, z)\right\} \nu(d z) D_{s+, z} \alpha d s\right] .
\end{aligned}
$$


Note by the definition of $Y$, with $Y(s)=Y^{\left(\beta_{\alpha}\right)}(s)$ and $s \geq t+h$, the process $Y(s)$ follows the dynamics

$$
d Y(s)=Y\left(s^{-}\right)\left[\frac{\partial b}{\partial x}(s) d s+\frac{\partial \sigma}{\partial x}(s) d^{-} B(s)+\int_{\mathbb{R}_{0}} \frac{\partial \theta}{\partial x}\left(s^{-}, z\right) \tilde{N}\left(d z, d^{-} s\right)\right],
$$

for $s, \geq t+h$ with initial condition $Y(t+h)$ in time $t+h$. By the Itô formula for forward integrals, this equation can be solved explicitly and we get

$$
Y(s)=Y(t+h) G(t+h, s), \quad s \geq t+h,
$$

where, in general, for $s \geq t$,

$$
\begin{aligned}
G(t, s):= & \exp \left(\int_{t}^{s}\left\{\frac{\partial b}{\partial x}(r)-\frac{1}{2}\left(\frac{\partial \sigma}{\partial x}\right)^{2}(r)\right\} d r+\int_{t}^{s} \frac{\partial \sigma}{\partial x}(r) d B^{-}(r)\right. \\
& +\int_{t}^{s} \int_{\mathbb{R}_{0}}\left\{\ln \left(1+\frac{\partial \gamma}{\partial x}(r, z)\right)-\frac{\partial \gamma}{\partial x}(r, z)\right\} \nu(d z) d t \\
& \left.+\int_{t}^{s} \int_{\mathbb{R}_{0}}\left\{\ln \left(1+\frac{\partial \gamma}{\partial x}\left(r^{-}, z\right)\right)\right\} \tilde{N}\left(d z, d^{-} r\right)\right) .
\end{aligned}
$$

Note that $G(t, s)$ does not depend on $h$, but $Y(s)$ does. Defining $H_{0}^{1}$ as in (3.8), it follows that

$$
A_{1}=E^{x}\left[\int_{t}^{T} \frac{\partial \widehat{H}_{0}^{1}}{\partial x}(s) Y(s) d s\right]
$$

Where $\widehat{H}_{0}^{1}(s)=H_{0}^{1}(s, \widehat{X}(s), \widehat{\pi}, \widehat{\theta})$.

Differentiating with respect to $h$ at $h=0$, we get

$$
\left.\frac{d}{d h} A_{1}\right|_{h=0}=\frac{d}{d h} E^{x}\left[\int_{t}^{t+h} \frac{\partial \widehat{H}_{0}^{1}}{\partial x}(s) Y(s) d s\right]_{h=0}+\frac{d}{d h} E^{x}\left[\int_{t+h}^{T} \frac{\partial \widehat{H}_{0}^{1}}{\partial x}(s) Y(s) d s\right]_{h=0} .
$$

Since $Y(t)=0$, we see that

$$
\frac{d}{d h} E^{x}\left[\int_{t}^{t+h} \frac{\partial H_{0}}{\partial x}(s) Y(s) d s\right]_{h=0}=0 .
$$

Therefore, by 6.15,

$$
\begin{aligned}
\left.\frac{d}{d h} A_{1}\right|_{h=0} & =\frac{d}{d h} E^{x}\left[\int_{t+h}^{T} \frac{\partial \widehat{H}_{0}^{1}}{\partial x}(s) Y(t+h) G(t+h, s) d s\right]_{h=0} \\
& =\int_{t}^{T} \frac{d}{d h} E^{x}\left[\frac{\partial \widehat{H}_{0}^{1}}{\partial x}(s) Y(t+h) G(t+h, s)\right]_{h=0} d s \\
& =\int_{t}^{T} \frac{d}{d h} E^{x}\left[\frac{\partial \widehat{H}_{0}^{1}}{\partial x}(s) G(t, s) Y(t+h)\right]_{h=0} d s
\end{aligned}
$$


where, $Y(t+h)$ is given by

$$
\begin{aligned}
Y(t+h)= & \int_{t}^{t+h} Y\left(r^{-}\right)\left[\frac{\partial b}{\partial x}(r) d r+\frac{\partial \sigma}{\partial x}(r) d^{-} B(r)+\int_{\mathbb{R}_{0}} \frac{\partial \gamma}{\partial x}\left(r^{-}, z\right) \tilde{N}\left(d z, d^{-} r\right)\right] \\
& +\alpha \int_{t}^{t+h}\left[\nabla_{\pi} b(r) d r+\nabla_{\pi} \sigma(r) d^{-} B(r)+\int_{\mathbb{R}_{0}} \nabla_{\pi} \gamma\left(r^{-}, z\right) \tilde{N}\left(d z, d^{-} r\right)\right] .
\end{aligned}
$$

Therefore, by the two preceding equalities,

$$
\left.\frac{d}{d h} A_{1}\right|_{h=0}=A_{1,1}+A_{1,2}
$$

where

$$
\begin{aligned}
A_{1,1} & =\int_{t}^{T} \frac{d}{d h} E^{x}\left[\frac { \partial H _ { 0 } } { \partial x } ( s ) G ( t , s ) \alpha \int _ { t } ^ { t + h } \left\{\nabla_{\pi} b(r) d r+\nabla_{\pi} \sigma(r) d^{-} B(r)\right.\right. \\
& \left.\left.+\int_{\mathbb{R}_{0}} \nabla_{\pi} \gamma\left(r^{-}, z\right) \widetilde{N}\left(d z, d^{-} r\right)\right\}\right]_{h=0} d s,
\end{aligned}
$$

and

$$
\begin{aligned}
A_{1,2} & =\int_{t}^{T} \frac{d}{d h} E^{x}\left[\frac { \partial H _ { 0 } } { \partial x } ( s ) G ( t , s ) \int _ { t } ^ { t + h } Y ( r ^ { - } ) \left\{\frac{\partial b}{\partial x}(r) d r+\frac{\partial \sigma}{\partial x}(r) d^{-} B(r)\right.\right. \\
& \left.\left.+\int_{\mathbb{R}_{0}} \frac{\partial \gamma}{\partial x}(r, z) \tilde{N}\left(d z, d^{-} r\right)\right\}\right]_{h=0} d s
\end{aligned}
$$

Applying again the duality formula, we have

$$
\begin{aligned}
A_{1,1}= & \int_{t}^{T} \frac{d}{d h} E^{x}\left[\alpha \int _ { t } ^ { t + h } \left\{\nabla_{\pi} b(r) F_{1}(t, s)+\nabla_{\pi} \sigma(r) D_{r} F_{1}(t, s)\right.\right. \\
& +F_{1}(t, s) D_{r^{+}} \nabla_{\pi} \sigma(r)+\int_{\mathbb{R}_{0}}\left\{\left(\nabla_{\pi} \gamma(r, z)+D_{r^{+}, z} \nabla_{\pi} \gamma(r, z)\right) D_{r, z} F_{1}(t, s)\right. \\
& \left.\left.\left.+D_{r^{+}, z} \nabla_{\pi} \gamma(r, z) F_{1}(t, s)\right\} \nu(d z)\right\} d r\right]_{h=0} d s \\
= & \int_{t}^{T} E^{x}\left[\alpha \left\{\left(\nabla_{\pi} b(t)+D_{t^{+}} \nabla_{\pi} \sigma(t)+\int_{\mathbb{R}_{0}} D_{t^{+}, z} \nabla_{\pi} \gamma(t, z) \nu(d z)\right) F_{1}(t, s)\right.\right. \\
& \left.\left.\nabla_{\pi} \sigma(t) D_{t} F_{1}(t, s)+\int_{\mathbb{R}_{0}}\left(\nabla_{\pi} \gamma(t, z)+D_{t^{+}, z} \nabla_{\pi} \gamma(t, z)\right) D_{t, z} F_{1}(t, s) \nu(d z)\right\}\right] d s,
\end{aligned}
$$

where we have put

$$
F_{1}(t, s)=\frac{\partial \widehat{H}_{0}^{1}}{\partial x}(s) G(t, s)
$$

Since $Y(t)=0$ we see that

$$
A_{1,2}=0 .
$$


We conclude that

$$
\begin{aligned}
\left.\frac{d}{d h} A_{1}\right|_{h=0} & =A_{1,1} \\
& =\int_{t}^{T} E\left[\alpha \left\{\left(\nabla_{\pi} b(t)+D_{t^{+}} \nabla_{\pi} \sigma(t)+\int_{\mathbb{R}_{0}} D_{t^{+}, z} \nabla_{\pi} \gamma(t, z) \nu(d z)\right) F_{1}(t, s)\right.\right. \\
& \left.\left.+\nabla_{\pi} \sigma(t) D_{t} F_{1}(t, s)+\int_{\mathbb{R}_{0}}\left(\nabla_{\pi} \gamma(t, z)+D_{t^{+}, z} \nabla_{\pi} \gamma(t, z)\right) D_{t, z} F_{1}(t, s) \nu(d z)\right\}\right] d s
\end{aligned}
$$

Moreover, we see that

$$
\begin{aligned}
\left.\frac{d}{d h} A_{2}\right|_{h=0}= & E\left[\left\{\widehat{K}_{1}(t)\left(\nabla_{\pi} b(t)+D_{t+} \nabla_{\pi} \sigma(t)+\int_{\mathbb{R}_{0}} D_{t+, z} \nabla_{\pi} \gamma(t, z) \nu(d z)\right)\right.\right. \\
& +\nabla_{\pi} f_{1}(t)+D_{t} \widehat{K}_{1}(t) \nabla_{\pi} \sigma(t) \\
& \left.\left.+\int_{\mathbb{R}_{0}} D_{t, z} \widehat{K}_{1}(t)\left(\nabla_{\pi} \gamma(t, z)+D_{t+, z} \nabla_{\pi} \gamma(t, z)\right) \nu(d z)\right\} \alpha\right], \\
\left.\frac{d}{d h} A_{4}\right|_{h=0}= & E\left[\widehat{K}_{1}(t) \nabla_{\pi} \sigma(t) D_{t+} \alpha\right], \\
\left.\frac{d}{d h} A_{6}\right|_{h=0}= & E\left[\int_{\mathbb{R}_{0}}\left\{\widehat{K}_{1}(t)+D_{t, z} \widehat{K}_{1}(t)\right\}\left(\nabla_{\pi} \gamma(t, z)+D_{t+, z} \nabla_{\pi} \gamma(t, z)\right) \nu(d z) D_{t+, z} \alpha\right] .
\end{aligned}
$$

On the other hand, differentiating $A_{3}$ with respect to $h$ at $h=0$, we get

$$
\begin{aligned}
\left.\frac{d}{d h} A_{3}\right|_{h=0}= & \frac{d}{d h} E\left[\int_{t}^{t+h} \widehat{K}_{1}(s) \frac{\partial \sigma(s)}{\partial x} D_{s+} Y(s) d s\right]_{h=0} \\
& +\frac{d}{d h} E\left[\int_{t+h}^{T} \widehat{K}_{1}(s) \frac{\partial \sigma(s)}{\partial x} D_{s+} Y(s) d s\right]_{h=0} .
\end{aligned}
$$

Since $Y(t)=0$, we see that

$$
\begin{aligned}
\left.\frac{d}{d h} A_{3}\right|_{h=0}= & \frac{d}{d h} E\left[\int_{t+h}^{T} \widehat{K}_{1}(s) \frac{\partial \sigma(s)}{\partial x} D_{s+}(Y(t+h) G(t+h, s)) d s\right]_{h=0} \\
= & \int_{t}^{T} \frac{d}{d h} E\left[\widehat{K}_{1}(s) \frac{\partial \sigma(s)}{\partial x} D_{s+}(Y(t+h) G(t+h, s))\right]_{h=0} d s \\
= & \int_{t}^{T} \frac{d}{d h} E\left[\widehat { K } _ { 1 } ( s ) \frac { \partial \sigma ( s ) } { \partial x } \left(D_{s+} G(t+h, s) \cdot Y(t+h)\right.\right. \\
& \left.\left.+D_{s+} Y(t+h) \cdot G(t+h, s)\right)\right]_{h=0} d s \\
= & \int_{t}^{T} \frac{d}{d h} E\left[\widehat{K}_{1}(s) \frac{\partial \sigma(s)}{\partial x} \cdot D_{s+} Y(t+h) G(t, s)\right]_{h=0} d s .
\end{aligned}
$$


Using the definition of $\widehat{p}$ and $\widehat{H}_{1}$ given respectively by 3.17 and 3.16 in the theorem, it follows by 6.13 that

$$
E\left[\nabla_{\pi} \widehat{H}_{1}(t, \widehat{X}(t), \widehat{u}(t)) \mid \mathcal{G}_{t}^{2}\right]+E[A]=0 \text { a.e. in }(t, \omega),
$$

where

$$
A=\left.\frac{d}{d h} A_{3}\right|_{h=0}+\left.\frac{d}{d h} A_{4}\right|_{h=0}+\left.\frac{d}{d h} A_{5}\right|_{h=0}+\left.\frac{d}{d h} A_{6}\right|_{h=0} .
$$

Similarly, we have

$$
\begin{aligned}
0= & \left.\frac{\partial}{\partial v} J_{2}(\widehat{\pi}, \widehat{\theta}+v \eta)\right|_{v=0} \\
= & E^{x}\left[\int _ { 0 } ^ { T } \int _ { \mathbb { R } _ { 0 } } \left\{\frac{\partial}{\partial x} f_{2}\left(t, \widehat{X}(t), \widehat{\pi}_{0}(t), \widehat{\pi}_{1}(t, z), \widehat{\theta}_{0}(t), \widehat{\theta}_{1}(t, z), z\right) \widehat{V}(t)\right.\right. \\
& \left.+\left.\nabla_{\pi} f_{1}\left(t, X^{(\widehat{\pi}, \theta)}(t), \widehat{\pi}_{0}(t), \widehat{\pi}_{1}(t, z), \theta_{0}(t), \theta_{1}(t, z), z\right)\right|_{\widehat{\theta}=\theta} \eta^{*}(t)\right\} \mu(d z) d t \\
& \left.+g^{\prime}(\widehat{X}(T)) \widehat{V}(t)\right]
\end{aligned}
$$

where

$$
\begin{aligned}
\widehat{V}(t)= & \widehat{V}_{\eta}(t)=\left.\frac{d}{d v} X^{(\widehat{\pi}, \widehat{\theta}+v \eta)}(t)\right|_{v=0} \\
& =\int_{0}^{t}\left\{\frac{\partial}{\partial x} b\left(s, \widehat{X}(s), \widehat{\pi}_{0}(s), \widehat{\theta}_{0}(s)\right) V(s)+\left.\nabla_{\pi} b\left(s, X^{\widehat{\pi}, \theta}(s), \widehat{\pi}_{0}(s), \theta_{0}(s)\right)\right|_{\theta=\widehat{\theta}} \eta^{*}(s)\right\} d s \\
& +\int_{0}^{t}\left\{\frac{\partial}{\partial x} \sigma\left(s, \widehat{X}(s), \widehat{\pi}_{0}(s), \widehat{\theta}_{0}(s)\right) V(s)+\left.\nabla_{\pi} \sigma\left(s, X^{\widehat{\pi}, \theta}(s), \widehat{\pi}_{0}(s), \theta_{0}(s)\right)\right|_{\theta=\widehat{\theta}} \eta^{*}(s)\right\} d B^{-}(s) \\
& +\int_{0}^{t} \int_{\mathbb{R}_{0}}\left\{\frac{\partial}{\partial x} \gamma\left(s, \widehat{X}\left(s^{-}\right), \widehat{\pi}_{0}\left(s^{-}\right), \widehat{\theta}_{0}\left(s^{-}\right), z\right) V(s)\right. \\
& \left.+\left.\nabla_{\pi} \gamma\left(s, X^{\widehat{\pi}, \theta}\left(s^{-}\right), \widehat{\pi}_{0}\left(s^{-}\right), \theta_{0}\left(s^{-}\right), z\right)\right|_{\theta=\widehat{\theta}} \eta^{*}(s)\right\} \widetilde{N}\left(d z, d^{-} s\right) .
\end{aligned}
$$

Define

$$
D(s)=D(t+h) G(t+h, s) ; s \geq t+h,
$$

where $G(t, s)$ is defined as in 3.20 . Using similar arguments, we get

$$
E\left[\nabla_{\pi} \widehat{H}_{2}(t, \widehat{X}(t), \widehat{u}(t)) \mid \mathcal{G}_{t}^{1}\right]+E[B]=0 \text { a.e. in }(t, \omega),
$$

where $B$ is given in the same way as $A$.

This completes the proof of (i).

(ii) Conversely, suppose that there exist $\widehat{\pi} \in \mathcal{A}_{\Pi}$ such that 3.14 holds. Then by reversing the previous arguments, we obtain that 6.13 holds for all $\beta_{\alpha}(s):=\alpha \chi_{[t, t+h]}\left(s \in \mathcal{A}_{\Pi}\right)$, 
where

$$
\begin{aligned}
A_{1}= & E^{x}\left[\int _ { t } ^ { T } \left\{\widehat{K}_{1}(t)\left(\frac{\partial b}{\partial x}(s)+D_{s+} \frac{\partial \sigma}{\partial x}(s)+\int_{\mathbb{R}_{0}} D_{s+, z} \frac{\partial \gamma}{\partial x}(s, z) \nu(d z)\right)+D_{t} \widehat{K}_{1}(t) \frac{\partial \sigma}{\partial x}(t)\right.\right. \\
& \left.\left.+\int_{\mathbb{R}_{0}} D_{s, z} \widehat{K}_{1}(t)\left(\frac{\partial \gamma}{\partial x}(s, z)+D_{s+, z} \frac{\partial \gamma}{\partial x}(s, z)\right) \nu(d z)\right\} Y^{\left(\beta_{\alpha}\right)}(s) d s\right], \\
A_{2}= & E^{x}\left[\int _ { t } ^ { t + h } \left\{\widehat{K}_{1}(t)\left(\nabla_{\pi} b(s)+D_{s+} \nabla_{\pi} \sigma(s)+\int_{\mathbb{R}_{0}} D_{t s, z} \nabla_{\pi} \gamma(t, z) \nu(d z)\right)+D_{t} \widehat{K}_{1}(t) \nabla_{\pi} \sigma(t)\right.\right. \\
& \left.\left.+\int_{\mathbb{R}_{0}} D_{s, z} \widehat{K}_{1}(t)\left(\nabla_{\pi} \gamma(s, z)+D_{s, z} \nabla_{\pi} \gamma(s, z)\right) \nu(d z)+\int_{\mathbb{R}_{0}} \nabla_{\pi} f_{1}(s, z) \mu(d z)\right\} \alpha d s\right], \\
A_{3}= & E^{x}\left[\int_{t}^{T} \widehat{K}_{1}(t) \frac{\partial \sigma(s)}{\partial x} D_{s+} Y^{\left(\beta_{\alpha}\right)}(s) d s\right], \\
A_{4}=+ & E\left[\int_{t}^{t+h} \widehat{K}_{1}(t) \nabla_{\pi} \sigma(s) D_{s+} \alpha d s\right], \\
A_{5}= & E^{x}\left[\int_{t}^{T} \int_{\mathbb{R}_{0}}\left(\widehat{K}_{1}(t)+D_{s, z} \widehat{K}_{1}(t)\right)\left\{\frac{\partial \gamma}{\partial x}(s)+D_{s+, z} \frac{\partial \gamma}{\partial x}(t)\right\} \nu(d z) D_{s+, z} Y^{\left(\beta_{\alpha}\right)}(s) d s\right], \\
A_{6}= & E^{x}\left[\int_{t}^{t+h} \int_{\mathbb{R}_{0}}\left(\widehat{K}_{1}(t)+D_{s, z} \widehat{K}_{1}(t)\right)\left\{\nabla_{\pi} \gamma(s, z)+D_{s+, z} \nabla_{\pi} \gamma(s, z)\right\} \nu(d z) D_{s+, z} \alpha d s\right],
\end{aligned}
$$

for some $t, h \in(0, T), t+h \leq T$, where $\alpha=\alpha(\omega)$ is bounded and $\mathcal{G}_{t}^{2}$-measurable. Hence, these equalities hold for all linear combinations of $\beta_{\alpha}$. Since all bounded $\beta \in \mathcal{A}_{\Pi}$ can be approximated pointwise boundedly in $(t, \omega)$ by such linear combinations, it follows that 6.13 holds for all bounded $\beta \in \mathcal{A}_{\Pi}$. Hence, by reversing the remaining part of the previous proof, we conclude that

$$
\left.\frac{\partial J_{1}}{\partial y}(\widehat{\pi}+y \beta, \widehat{\theta})\right|_{y=0}=0, \text { for all } \beta .
$$

Similarly, suppose that there exist $\hat{\theta} \in \mathcal{A}_{\Theta}$ such that 3.15 holds. Then, the above argument leads us to conclude that

$$
\left.\frac{\partial J_{2}}{\partial v}(\widehat{\pi}, \widehat{\theta}+v \eta)\right|_{v=0}=0, \text { for all } \eta
$$

On the other hand, assume moreover that $\pi \rightarrow J_{1}(\pi, \widehat{\theta})$, then

$$
\begin{aligned}
0=\frac{1}{y}\left(J_{1}(\pi+y \beta, \widehat{\theta})-J_{1}(\pi, \widehat{\theta})\right) & =\frac{1}{y}\left(J_{1}\left(1-y \frac{\pi}{1-y}+y \beta, \widehat{\theta}\right)-J_{1}(\pi, \widehat{\theta})\right) \\
& \geq \frac{1}{y}\left((1-y) J_{1}\left(\frac{\pi}{1-y}, \widehat{\theta}\right)+y J_{1}(\beta, \widehat{\theta})-J_{1}(\pi, \widehat{\theta})\right) \\
& =\frac{1}{y}\left(J_{1}\left(\frac{\pi}{1-y}, \widehat{\theta}\right)-J_{1}(\pi, \widehat{\theta})\right)+J_{1}(\beta, \widehat{\theta})-J_{1}\left(\frac{\pi}{1-y}, \widehat{\theta}\right) .
\end{aligned}
$$


Taking the limit for $y \rightarrow 0$, and using the fact that $\lim _{y \rightarrow 0} \frac{1}{y}\left(J_{1}\left(\frac{\pi}{1-y}, \widehat{\theta}\right)-J_{1}(\pi, \widehat{\theta})\right)=0$, we obtain that $0 \geq J_{1}(\beta, \widehat{\theta})-J_{1}(\widehat{\pi}, \widehat{\theta})$. Since $\beta$ can be chosen within the set $\mathcal{A}_{\pi}$, we obtain by formally setting $\beta=\pi$ that

$$
J_{1}(\pi, \widehat{\theta}) \leq J_{1}(\widehat{\pi}, \widehat{\theta}) \text { for all } \pi \in \mathcal{A}_{\pi}
$$

Analogously, we obtain

$$
J_{2}(\widehat{\pi}, \theta) \leq J_{1}(\widehat{\pi}, \widehat{\theta}) \text { for all } \theta \in \mathcal{A}_{\theta}
$$

This means that $(\widehat{\pi}, \widehat{\theta})$ is a Nash-equilibrium for the market. is concave in each $\pi$ or $\theta$ This complete the proof. 